\title{
Consequences of $\mu-\tau$ reflection symmetry at DUNE
}

\author{
Newton Nath ${ }^{1,2, *}$ \\ ${ }^{1}$ Institute of High Energy Physics, Chinese Academy of Sciences, Beijing 100049, China \\ ${ }^{2}$ School of Physical Sciences, University of Chinese Academy of Sciences, Beijing 100049, China
}

(Received 4 June 2018; published 17 October 2018)

\begin{abstract}
We consider minimal type-I seesaw framework to realize $\mu-\tau$ reflection symmetry in the low-energy neutrino mass matrix, $M_{\nu}$. Considering DUNE experiment, we scrutinize its potential to measure the precision of 2-3 mixing angle, $\theta_{23}$ and the Dirac $C P$-phase, $\delta$ for the given symmetry. Later, we examine the precision of these two parameters considering NuFit-3.2 data as one of the important true points. To study the low-energy phenomenology, we further discuss various breaking patterns of such an exact symmetry. Moreover, for each breaking scenario we perform the capability test of DUNE for the determination of $\theta_{23}$ and to establish the phenomenon of $C P$ violation considering the true benchmark point arising from the breaking of $\mu-\tau$ reflection symmetry. We also make remarks on the potential of DUNE to rule out maximal $C P$-violation or $C P$-conservation hypothesis at a certain confidence level for different scenarios.
\end{abstract}

DOI: 10.1103/PhysRevD.98.075015

\section{INTRODUCTION}

During last a few years, there has been remarkable progress in the field of neutrino physics which guided us to understand some intriguing aspects of neutrinos in a comprehensive manner. It is now a well established phenomenon from different experimental results that neutrinos possess nonzero mass, and their different flavors are mixed [1]. However, the dynamical origin associated with neutrinos mass generation as well as mixing patterns are still unknown. There have been numerous theoretical attempts to understand the nature of tiny neutrino masses, among which the seesaw mechanism is considered to be the highly appreciated one [2-6]. The simplest way to generate neutrino masses is to add at least two $S U(2)$ singlet righthanded neutrino fields (i.e., $N_{\mu R}, N_{\tau R}$ ) in the Standard Model (SM). The relevant SM gauge invariant Lagrangian containing the neutrino Yukawa matrix and the Majorana neutrino mass matrix can be written as

$$
-\mathcal{L} \supset \bar{L}_{\alpha L} Y_{\nu} N_{R} \tilde{H}+\frac{1}{2} \overline{N_{R}^{C}} M_{R} N_{R}+\text { H.c. }
$$

where $L_{\alpha L}=\left(\nu_{\alpha}, \alpha\right)_{L}^{T}$ is the left-handed lepton doublet, $Y_{\nu}$ denotes the neutrino Yukawa matrix, $\tilde{H}=i \sigma_{2} H^{*}$ with $H$ being the Higgs doublet in the SM. Also, $M_{R}$ is the

\footnotetext{
*newton@ihep.ac.cn
}

Published by the American Physical Society under the terms of the Creative Commons Attribution 4.0 International license. Further distribution of this work must maintain attribution to the author(s) and the published article's title, journal citation, and DOI. Funded by SCOAP ${ }^{3}$.
Majorana neutrino mass matrix and $C$ denotes the charge-conjugation operator. After spontaneous symmetry breaking, one obtains the Dirac neutrino mass term as $\bar{\nu}_{\alpha L} M_{D} N_{R}+$ H.c., where $M_{D}=v Y_{\nu}$ is the Dirac neutrino mass matrix with vacuum expectation value (vev), $v=$ $\langle H\rangle \approx 174 \mathrm{GeV}$ [1]. Employing seesaw mechanism, one gets light neutrino mass matrix in type-I seesaw formalism as, $M_{\nu} \approx-M_{D} M_{R}^{-1} M_{D}^{T}$ and diagonalization of such $M_{\nu}$ leads to three active neutrino masses $m_{i}$ (for $i=1,2,3$ ).

Furthermore, flavor symmetry based approaches have received numerous attention to explain the observed neutrino mixing patterns as discussed in Refs. [7-11] and the references therein. Among number of such approaches, $\mu-\tau$ reflection symmetry attracts a lot of attention in recent times which was originally discussed in Ref. [12] (see Ref. [13] for a latest review). This symmetry predicts: the maximal atmospheric mixing angle $\theta_{23}$, i.e., $\theta_{23}=$ $45^{\circ}$ along with the maximal value of Dirac $C P$ phase $\delta$, i.e., $\delta= \pm 90^{\circ}$; and trivial values for the two Majorana phases with nonzero $\theta_{13}$. Indeed, in recent times there are many attempts toward $\mu-\tau$ reflection symmetry as outlined in Refs. [14-32].

In this work, we embed $\mu-\tau$ reflection symmetry in minimal type-I seesaw formalism such that one can address both neutrino masses and mixing patterns (see Ref. [33] for recent review). Later, we study its consequences considering next-generation super beam Deep Underground Neutrino Experiment (DUNE). This statistically high potential experiment will improve the precision of the atmospheric mixing angle, $\theta_{23}$ and play a key role to probe the leptonic Dirac $C P$ violating phase, $\delta$ [34]. Because of this, DUNE can test various flavor symmetry models and helps us to understand some inherent physics associate with it. 
At the given framework along with maximal $\delta$ and $\theta_{23}$, we also find remaining oscillation parameters both analytically as well as numerically. Considering this as a true benchmark point, we depict the allowed area in the $\left(\delta-\sin ^{2} \theta_{23}\right)$ plane for DUNE at various confidence levels which serves our intention to inspect precision of these two lesser known parameters. This also show the potential of DUNE to know how well it can measure $\delta$ and $\theta_{23}$. Moreover, latest results of global-fit of neutrino oscillation data from NuFit-3.2 collaboration [35,36] favors higher octant of $\theta_{23}$ along with nonmaximal $\delta .{ }^{1}$ Also, results of ongoing neutrino oscillation experiments (e.g., T2K [37] and $\mathrm{NO} \nu \mathrm{A}$ [38]) are in well agreement with the predictions of the concerned symmetry but, still show large uncertainties in their measurement of $\delta$ and $\theta_{23}$. Therefore, it is tenacious to accept the exact nature of $\mu-\tau$ reflection symmetry. In that respect, it is worthwhile to study various broken scenarios of such a symmetry. To proceed with phenomenological study, we first perform our analysis considering global best-fit values as our benchmark point $[35,36]$. Afterwards, we consider breaking of $\mu-\tau$ reflection symmetry by introducing explicit breaking parameter in the high-energy neutrino mass matrices $M_{D}, M_{R}$, respectively. ${ }^{2}$ For each scenario, we find the set of neutrino oscillation parameters and perform the capability test of DUNE in the $\left(\delta-\sin ^{2} \theta_{23}\right)$ plane. Considering different cases, we analyze the potential of DUNE to rule out the possibility of maximal $C P$-violation (CPV) as well as $C P$ conservation hypothesis at a given confidence level. Some recent studies considering different flavor models in the context of long baseline experiments have been performed in $[31,39-48]$.

We organize rest of the paper as follows. In Sec. II, we present a general setup of the $\mu-\tau$ reflection symmetry and perform our analysis in the given scenario for DUNE. We also present our numerical details in this section.
We proceed to discuss our results considering NuFit-3.2 data in Sec. III. Furthermore, in subsequent subsections of Sec. III, we discuss the breaking of $\mu-\tau$ reflection symmetry by introducing explicit breaking parameter in $M_{D}$ and $M_{R}$, respectively, and their implications in the context of DUNE. Finally, we summarize our noteworthy results in Sec. IV.

\section{PHENOMENOLOGY AT $\mu-\tau$ REFLECTION SYMMETRY}

The $\mu-\tau$ reflection symmetry at the low-energy neutrino mass matrix, $M_{\nu}$ was first proposed in Ref. [12] which leads us to following four predictions,

$$
\begin{array}{ll}
M_{e e}=M_{e e}^{*}, & M_{\mu \tau}=M_{\mu \tau}^{*}, \\
M_{e \mu}=M_{e \tau}^{*}, & M_{\mu \mu}=M_{\tau \tau}^{*},
\end{array}
$$

where $M_{\alpha \beta}$, (with $\left.\alpha, \beta=e, \mu, \tau\right)$ are the elements of $M_{\nu}$. We consider minimal type-I seesaw mechanism to realize $\mu-\tau$ reflection symmetry at $M_{\nu}$. To achieve such symmetry, we extend the SM fields content by adding two right-handed neutrino fields which are singlet under the SM gauge group. Without loss of generality, we consider the following texture of $M_{D}$ to realize $\mu-\tau$ reflection symmetry,

$$
M_{D}=\left(\begin{array}{cc}
a & a^{*} \\
b & c \\
c^{*} & b^{*}
\end{array}\right)=\left(\begin{array}{cc}
a e^{i \phi_{a}} & a e^{-i \phi_{a}} \\
b e^{i \phi_{b}} & c e^{i \phi_{c}} \\
c e^{-i \phi_{c}} & b e^{-i \phi_{b}}
\end{array}\right)
$$

Also, we adopt diagonal $M_{R}$ of the form $M_{R}=$ $\operatorname{diag}\left(M_{1}, M_{1}\right)$ with degenerate heavy Majorana neutrino masses. ${ }^{3}$ Further, considering type-I seesaw mechanism, we obtain the effective neutrino mass matrix for the light neutrinos as

$$
-M_{\nu}=M_{D} M_{R}^{-1} M_{D}^{T},=\frac{1}{M_{1}}\left(\begin{array}{ccc}
2 a^{2} \cos 2 \phi_{a} & a b e^{i\left(\phi_{a}+\phi_{b}\right)}+a c e^{-i\left(\phi_{a}-\phi_{c}\right)} & a b e^{-i\left(\phi_{a}+\phi_{b}\right)}+a c e^{i\left(\phi_{a}-\phi_{c}\right)} \\
- & b^{2} e^{2 i \phi_{b}}+c^{2} e^{2 i \phi_{c}} & 2 b c \cos \left(\phi_{b}-\phi_{c}\right) \\
- & - & b^{2} e^{-2 i \phi_{b}}+c^{2} e^{-2 i \phi_{c}}
\end{array}\right) .
$$

We notice that the elements of $M_{\nu}$ as given by Eq. (4) satisfy all the conditions of Eq. (2) and hence leads to $\mu-\tau$ reflection symmetry. ${ }^{4}$ In the standard PDG [1] parametrization, the unitary mixing matrix which diagonalizes neutrino mass matrix, $M_{\nu}$, can be written as,

\footnotetext{
${ }^{1}$ Note that $\theta_{23}<45^{\circ}$ is called a lower octant (LO), where $\theta_{23}>45^{\circ}$ is called a higher octant (HO).

${ }^{2}$ Authors in Refs. [24,29] have also studied the breaking of such symmetry considering renormalization group (RG) running for different neutrino mass textures in details. However, the impact of breaking due to RG running is very mild; hence, we do not consider this.

${ }^{3}$ It is possible to find the considered mass textures using a suitable flavor group along with preferred $\mathbb{Z}_{n}$ cyclic group. As our intention is to study the impact of these textures rather their theoretical origin, hence we do not perform this study here.

${ }^{4}$ Note that nondegenerate Majorana neutrino mass matrix does not satisfy all the conditions mentioned in Eq. (2) and thus does not lead to the concerned symmetry which we discuss in Sec. III.
} 


$$
V=P_{l} U P_{\nu},=P_{l}\left(\begin{array}{ccc}
c_{12} c_{13} & s_{12} c_{13} & s_{13} e^{-\mathrm{i} \delta} \\
-s_{12} c_{23}-c_{12} s_{13} s_{23} e^{\mathrm{i} \delta} & c_{12} c_{23}-s_{12} s_{13} s_{23} e^{\mathrm{i} \delta} & c_{13} s_{23} \\
s_{12} s_{23}-c_{12} s_{13} c_{23} e^{\mathrm{i} \delta} & -c_{12} s_{23}-s_{12} s_{13} c_{23} e^{\mathrm{i} \delta} & c_{13} c_{23}
\end{array}\right) P_{\nu}
$$

where $c_{i j}=\cos \theta_{i j}, s_{i j}=\sin \theta_{i j}$ (for $i<j=1,2,3$ ). Here, $P_{l}$ contains three unphysical phases of the form $P_{l}=$ $\operatorname{diag}\left(e^{i \phi_{e}}, e^{i \phi_{\mu}}, e^{i \phi_{\tau}}\right)$ which can be absorbed by the rephasing of charged lepton fields, where $P_{\nu}=\operatorname{diag}\left(e^{i \rho}, e^{i \sigma}, 1\right)$ contains two Majorana phases.

With the above form of $M_{\nu}$ as given by Eq. (4), one can find that there exist six predictions for the leptonic mixing angles and phases which are ${ }^{5}$

$$
\phi_{e}=90, \quad \phi_{\mu}=-\phi_{\tau}=\phi, \quad \theta_{23}=45^{\circ}, \quad \delta= \pm 90^{\circ}, \quad \rho, \quad \sigma=0^{\circ} \quad \text { or } \quad 90^{\circ} .
$$

Note that under $\mu-\tau$ reflection symmetry the value of $\theta_{13}, \theta_{12}$ remain unspecified. Also it does not shed any light on the nature of possible neutrino mass ordering i.e., whether neutrino masses obey normal mass ordering (NMO, $m_{3}>m_{2}>m_{1}$ ) or inverted mass ordering (IMO, $m_{3}<m_{1} \approx m_{2}$ ). We find the analytical form of the mixing angles and masses in terms of model parameters as

$$
\begin{aligned}
& \theta_{13}=\mp \tan ^{-1}\left[\frac{b^{2} \sin 2 \varphi_{b}+c^{2} \sin 2 \varphi_{c}}{a\left(b \sin \varphi_{a b}+c \sin \varphi_{a c}\right)}\right], \\
& \theta_{12}= \begin{cases}\frac{1}{2} \tan ^{-1}\left[\frac{2 \sqrt{2} a \cos 2 \theta_{13}\left(b \sin \varphi_{a b}+c \sin \varphi_{a c}\right)}{c_{13}\left[\left(b^{2} \cos 2 \varphi_{b}+c^{2} \cos 2 \varphi_{c}-2 b c \cos \varphi_{b c}\right) \cos 2 \theta_{13}-\left(b^{2} \cos 2 \varphi_{b}+c^{2} \cos 2 \varphi_{c}+2 b c \cos \varphi_{b c}\right) s_{13}^{2}+2 a^{2} \cos 2 \phi_{a} c_{13}^{2}\right]}\right] ; & \text { for NMO } \\
\frac{1}{2} \tan ^{-1}\left[\frac{2 \sqrt{2} a\left(b \sin \varphi_{a b}+c \sin \varphi_{a c}\right) s_{13}^{2}}{c_{13}\left[\left(b^{2} \cos 2 \varphi_{b}+c^{2} \cos 2 \varphi_{c}\right)\left(1+s_{13}^{2}\right)+2 c_{13}^{2} b c \cos \varphi_{b c}\right]}\right] ; & \text { for IMO }\end{cases}
\end{aligned}
$$

where $\varphi_{b, c}=\left(\phi-\phi_{b, c}\right), \varphi_{a b, c}=\left(\phi-\phi_{a}-\phi_{b, c}\right), \varphi_{b c}=$ $-\left(\phi_{b}-\phi_{c}\right)$.

Similarly, one can calculate masses of light neutrinos by diagonalizing $M_{\nu}$ of Eq. (4) as

$$
V^{\dagger} M_{\nu} V^{*}=\operatorname{diag}\left(m_{1}, m_{2}, m_{3}\right)
$$

where $m_{i}$ 's $(i=1,2,3)$ are the active neutrino masses. Further, the masses can be expressed for NMO as

$$
\begin{aligned}
m_{1}= & 0 \\
\tilde{m}_{2}= & \frac{2 \sqrt{2} a\left(b \sin \varphi_{a b}+c \sin \varphi_{a c}\right)}{c_{13} \sin 2 \theta_{12} M_{1}}, \\
m_{3}= & \frac{1}{M_{1}}\left[4 b c \cos \varphi_{b c}+2 a^{2} \cos 2 \phi_{a}\right. \\
& \left.+\frac{2 \sqrt{2} a\left(b \sin \varphi_{a b}+c \sin \varphi_{a c}\right)}{c_{13} \sin 2 \theta_{12}}\right],
\end{aligned}
$$

whereas, expressions for IMO can be written as

\footnotetext{
${ }^{5}$ For a detailed discussion on the adopted phase conventions see appendix of Ref. [29].
}

$$
\begin{aligned}
m_{1}= & \frac{1}{M_{1}}\left[2 b c \cos \varphi_{b c}-a^{2} \cos 2 \phi_{a}\right. \\
& \left.-\frac{2 \sqrt{2} a\left(b \sin \varphi_{a b}+c \sin \varphi_{a c}\right)}{c_{13} \sin 2 \theta_{12}}\right], \\
\tilde{m}_{2}= & \frac{1}{M_{1}}\left[-2 b c \cos \varphi_{b c}-a^{2} \cos 2 \phi_{a}\right. \\
& \left.+\frac{2 \sqrt{2} a\left(b \sin \varphi_{a b}+c \sin \varphi_{a c}\right)}{c_{13} \sin 2 \theta_{12}}\right], \\
m_{3}= & 0 .
\end{aligned}
$$

Here, $\tilde{m}_{2}=m_{2} e^{2 i \sigma}$ and $\sigma$ can take value either $0^{\circ}$ or $90^{\circ}$. Also note that as the minimal seesaw formalism always predicts massless lightest neutrino, one has the freedom of eliminating one of the Majorana phases. Thus, in this study we do not consider phase, $\rho$.

To proceed further and to investigate low-energy phenomenology, we first give here simulation and experimental details that are considered in this work. The principle strategy of our numerical analysis is to scan all the highenergy variables of $Y_{\nu}$ and $M_{R}$ as free variables and later constrain the allowed space of high-energy variables to find neutrino oscillation parameters which are compatible with the latest NuFit-3.2 data [35,36] at low energies. We vary different parameters as, 


$$
\begin{aligned}
|a|,|b|,|c| & \in[0,1] v, \quad \phi_{a, b, c} \in\left[0,360^{\circ}\right), \\
M_{1} & \in\left[10^{12}, 10^{15}\right] \mathrm{GeV} .
\end{aligned}
$$

We use the nested sampling package Multinest [49-51] to guide the parameter scan with the built $\chi^{2}$ function considering latest NuFit-3.2 data [35,36]. The analytical expression of the Gaussian- $\chi_{\min }^{2}$ function that we use in our numerical simulation is defined as,

$$
\chi_{\min }^{2}=\min \sum_{i} \frac{\left[\xi_{i}^{\text {True }}-\xi_{i}^{\text {Test }}\right]^{2}}{\sigma\left[\xi_{i}^{\text {True }}\right]^{2}},
$$

where $\xi=\left\{\theta_{12}, \theta_{13}, \theta_{23}, \Delta m_{21}^{2},\left|\Delta m_{31}^{2}\right|\right\}$, represents the set of neutrino oscillation parameters. Here, $\xi_{i}^{\text {Ture }}$ represent the current best-fit values of the latest NuFit-3.2 data [35,36] and $\xi_{i}^{\text {Test }}$ correspond to the predicted values for a given set of parameters in theory. We also symmetrize standard deviation, $\sigma\left[\xi_{i}^{\text {True }}\right]$ considering $1 \sigma$ errors as given by Ref. [35,36].

We consider here DUNE, which is a proposed next generation superbeam experiment at Fermilab, USA [34,52] designing to detect neutrinos. This experiment will utilize existing NuMI (Neutrinos at the Main Injector) beamline design at Fermilab as a neutrino source. The far detector of DUNE will be placed at Sanford Underground Research Facility (SURF) in Lead, South Dakota, at a distance of $1300 \mathrm{~km}$ (800 mile) from neutrino source. DUNE collaboration has planned to use LArTPC (liquid argon time-projection chamber) detector. For the numerical simulation of the DUNE data, we use the GLOBES package $[53,54]$ along with the required auxiliary files presented in Ref. [52]. We perform our simulation considering 40 kton fiducial mass far detector. We also consider the flux corresponding to $1.07 \mathrm{MW}$ beam power which gives $1.47 \times 10^{21}$ protons on target (POT) per year due to $80 \mathrm{GeV}$ proton beam energy. In addition, we adopt signal and background normalization uncertainties for appearance as well as disappearance channel as presented in DUNE CDR [52]. Further, we distribute the total exposure of DUNE (i.e., 300 kton-MWyears) in two scenarios: (i) In the first scenario, we perform our analysis only with neutrino mode considering 7 years of neutrino run, i.e., DUNE $[7 \nu+0 \bar{\nu}]$, and (ii) in the second scenario, we consider 3.5 years each of the neutrino and antineutrino mode i.e., DUNE $[3.5 \nu+3.5 \bar{\nu}]$. We also add $5 \%$ prior on $\sin ^{2} 2 \theta_{13}$ in our analysis.

The main steps to carry out our numerical analysis are to calculate a set of neutrino oscillation parameters corresponding to the minimum $\chi^{2}\left(=\chi_{\min }^{2}\right)$, as defined by Eq. (12), using Multinest in this model. Later, considering this set of parameters as a true benchmark value, we generate DUNE results using GLOBES and present the allowed parameter space in the test $\left(\delta-\sin ^{2} \theta_{23}\right)$ plane. We utilize the GLOBES inbuilt $\chi^{2}$ function for the data analysis. In this study, we marginalize all the oscillation parameters over their $3 \sigma$ range as given by Table II. In addition, we marginalize $\delta$ in the range $\delta \in\left[0^{\circ}, 360^{\circ}\right)$ for each scenario unless otherwise stated.

In Fig. 1, we present our results in the framework of $\mu-\tau$ reflection symmetry. We calculate the numerical values for the set of neutrino oscillation parameters in the given scenario corresponding to $\chi_{\min }^{2}$ as given in Table I. Considering this true set of parameters, we find the allowed area in the $\left(\delta-\sin ^{2} \theta_{23}\right)$ plane in the case of DUNE which we have depicted in Fig. 1. The green-, pink-, and blue-colored contours represent $1 \sigma, 3 \sigma$, and $5 \sigma$ allowed parameter space, respectively, and the red-star point represents the true value of $\left(\delta, \sin ^{2} \theta_{23}\right)$. Further, the top and bottom row show our results for DUNE $[7 \nu+0 \bar{\nu}]$ and DUNE $[3.5 \nu+3.5 \bar{\nu}]$ respectively. ${ }^{6}$ Also, the vertical black-dashed lines represent maximal CPV corresponding to $\delta=90^{\circ}$ and $270^{\circ}$, respectively. Similarly, the blue-dotted line signifies the $C P$-conserving value $\delta=180^{\circ}$, and horizontal black-dashed line represents $\sin ^{2} \theta_{23}=0.5$. Note that we consider similar color details throughout this work.

Considering maximal value of $\left(\delta, \sin ^{2} \theta_{23}\right)$ as a true benchmark point, we notice from the first row of Fig. 1 that 7 years of neutrino run of DUNE can rule out $C P$ conservation hypothesis at $1 \sigma$ C.L. for both the mass ordering (i.e., NMO, IMO) as shown by green contour. This observation remains true even at $3 \sigma$ C.L. for both the mass ordering as presented in the pink contour. To justify this point, we notice from upper panel that the pink contour does not intersect with the vertical blue-dotted line which provides clear evidence of the ruling out of $\mathrm{CP}$ conservation hypothesis at the same confidence level. Besides this, we notice from the $5 \sigma$ contour (see blue contour) that DUNE cannot exclude $C P$-conservation hypothesis for both the mass orderings. In addition, we also notice that the precision of $C P$-phase, $\delta$ is marginally better in the case of IMO compared to NMO, where $\sin ^{2} \theta_{23}$ shows almost similar precision for both cases at $5 \sigma$ C.L. From the second row of Fig. 1, we notice that DUNE can rule out the $C P$-conservation hypothesis even at $5 \sigma$ C.L. for IMO (see right panel), where in the case NMO, it can almost exclude the same except for some regions around $\left(\delta=0^{\circ} / 360^{\circ}, \sin ^{2} \theta_{23}=0.5\right)$. Finally, we notice from the top row that DUNE can rule out one half-plane of $\delta$ at $3 \sigma$ C.L., but at $5 \sigma$ C.L., it can exclude almost the same for both the mass orderings. In the case of $\mathrm{NMO}$ (for true $\delta=90^{\circ}$ ), we observe that DUNE can rule out $\delta$ in the range $\delta \in\left[180^{\circ}, 360^{\circ}\right]$, whereas for IMO (for true $\delta=270^{\circ}$ ), it can rule out $\delta$ in the range $\delta \in\left[0^{\circ}, 180^{\circ}\right]$ at $3 \sigma$ C.L. Similarly, from the bottom row, we notice that the same conclusion remains true even at $5 \sigma$ C.L. except for small regions for NMO.

\footnotetext{
${ }^{6}$ Note that authors of Ref. [55] have performed a detailed analysis on the sensitivity of these poorly known parameters considering various combinations of $(\nu+\bar{\nu})$ for DUNE.
} 

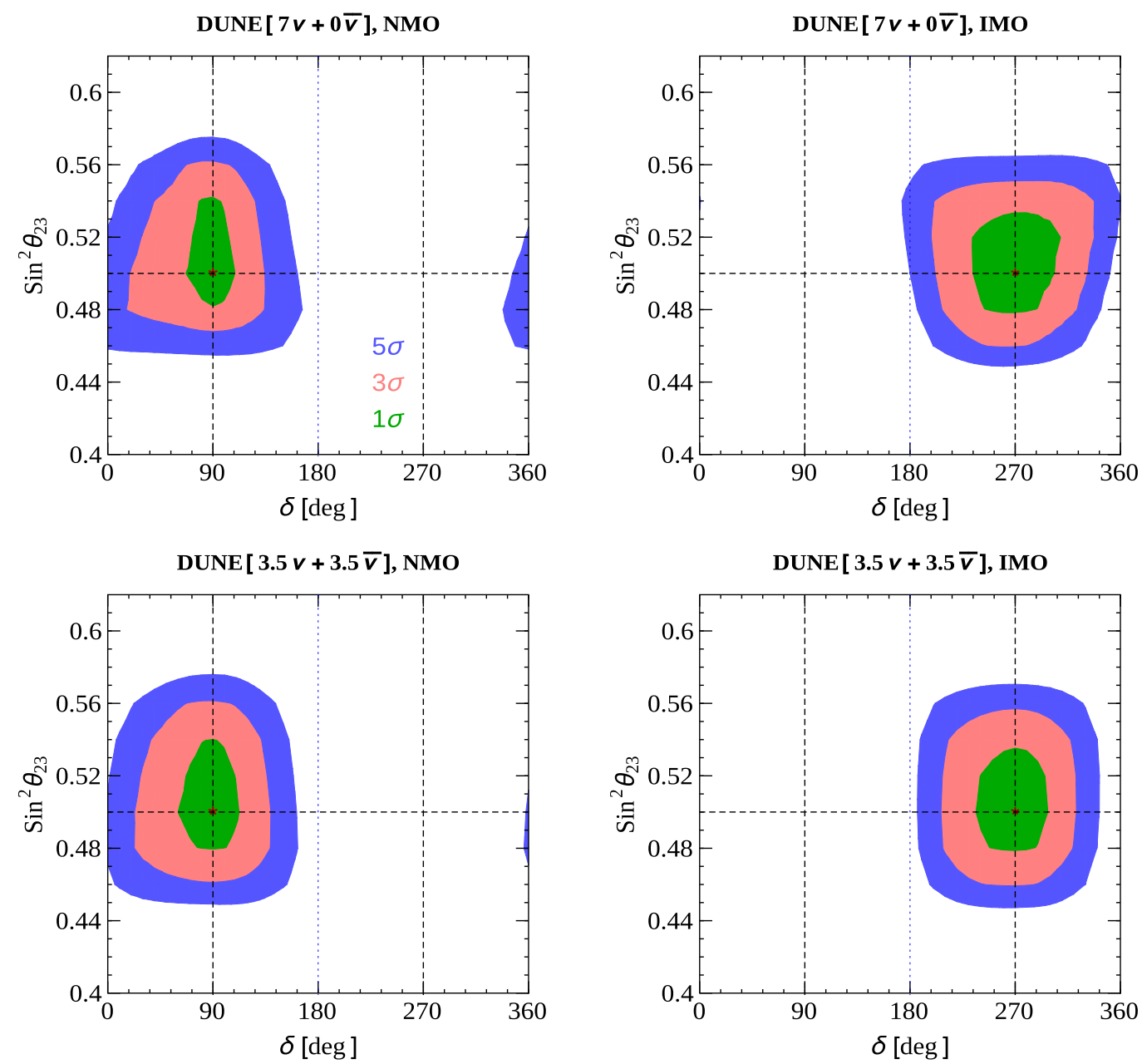

FIG. 1. Allowed parameter space of DUNE in the $\left(\delta-\sin ^{2} \theta_{23}\right)$ plane in the $\mu-\tau$ reflection symmetry scenario. Here, the green, pink, and blue colors represent $1 \sigma, 3 \sigma$, and $5 \sigma$ allowed contours and red-* signifies the true value of $\left(\delta, \sin ^{2} \theta_{23}\right)$. Also, the left (right) column represents normal (inverted) mass ordering and the top (bottom) row shows our results for DUNE[7 $2 \nu+0 \bar{\nu}](\mathrm{DUNE}[3.5 \nu+3.5 \bar{\nu}])$.

Having discussed our results in the $\mu-\tau$ reflection symmetry scenario considering DUNE, in the following section, we proceed to perform our analysis by utilizing current oscillations data. Later, we also examine different symmetry breaking scenarios where we will discuss the impact of breaking parameter on the poorly measured parameters, $\delta$ and $\sin ^{2} \theta_{23}$.

TABLE I. Set of neutrino oscillation parameters at $\chi_{\min }^{2}=0.10$ $\left(\chi_{\min }^{2}=0.82\right)$ for $\mathrm{NMO}(\mathrm{IMO})$ in the $\mu-\tau$ reflection symmetry scenario.

\begin{tabular}{lcc}
\hline \hline Parameters & NMO $\left(\chi_{\min }^{2}=0.10\right)$ & IMO $\left(\chi_{\min }^{2}=0.82\right)$ \\
\hline$\Delta \mathrm{m}_{21}^{2}\left[10^{-5} \mathrm{eV}^{2}\right]$ & 7.401 & 7.50 \\
$\left|\Delta \mathrm{m}_{31}^{2}\right|\left[10^{-3} \mathrm{eV}^{2}\right]$ & 2.498 & 2.465 \\
$\sin ^{2} \theta_{12}$ & 0.304 & 0.303 \\
$\sin ^{2} \theta_{23}$ & 0.50 & 0.50 \\
$\sin ^{2} \theta_{13}$ & 0.02217 & 0.02218 \\
$\delta$ [deg] & 90 & 270 \\
\hline \hline
\end{tabular}

\section{PHENOMENOLOGY BEYOND $\mu-\tau$ REFLECTION SYMMETRY}

As the current best-fit value of neutrino oscillation data prefers nonmaximal value of $\delta, \sin ^{2} \theta_{23}$, we start our discussion considering this as our true benchmark point in this section. Furthermore, in subsequent subsections, we perform our study considering different breaking scenarios of $\mu-\tau$ reflection symmetry and its impact in the context of DUNE.

\section{A. Analysis of global best-fit data}

In Table II, we give the latest results of global-fit of neutrino oscillation data as obtained by NuFit-3.2 [35] collaboration. We notice from the table that the best-fit points of latest analysis favor higher octant for the 2-3 mixing angle, $\theta_{23}$ and nonmaximal value for the Dirac $C P$ phase, $\delta$ for both the mass orderings.

In Fig. 2, we present our results in the $\left(\delta-\sin ^{2} \theta_{23}\right)$ plane for DUNE considering best-fit values of NuFit-3.2 data as 
TABLE II. The best-fit values and $3 \sigma$ range of neutrino oscillation parameters [35].

\begin{tabular}{llcc}
\hline \hline $\begin{array}{l}\text { Oscillation } \\
\text { parameters }\end{array}$ & $\begin{array}{c}\text { NMO } \\
\text { best-fit }\end{array}$ & $\begin{array}{c}\text { IMO } \\
\text { best-fit }\end{array}$ & \multicolumn{1}{c}{ Any ordering $3 \sigma$} \\
\hline$\Delta \mathrm{m}_{21}^{2}\left[10^{-5} \mathrm{eV}^{2}\right]$ & 7.40 & 7.40 & $6.80 \rightarrow 8.02$ \\
$\left|\Delta \mathrm{m}_{31}^{2}\right|\left[10^{-3} \mathrm{eV}^{2}\right]$ & 2.494 & 2.465 & $2.399 \rightarrow 2.593(\mathrm{NMO})$ \\
& & & $2.395 \rightarrow 2.536(\mathrm{IMO})$ \\
$\sin ^{2} \theta_{12}$ & 0.307 & 0.307 & $0.272 \rightarrow 0.346$ \\
$\sin ^{2} \theta_{23}$ & 0.538 & 0.554 & $0.418 \rightarrow 0.613$ \\
$\sin ^{2} \theta_{13}$ & 0.02206 & 0.02227 & $0.019 \rightarrow 0.0243$ \\
$\delta[\mathrm{deg}]$ & 234 & 278 & $144 \rightarrow 374$ \\
\hline \hline
\end{tabular}

our true benchmark point. Here red-star represents true value of $\left(\delta, \sin ^{2} \theta_{23}\right)$ i.e., $\left(234^{\circ}, 0.538\right)$ and $\left(278^{\circ}, 0.554\right)$ corresponding to NMO and IMO, respectively. From first plot of top row, we notice that DUNE can exclude the possibility of having maximal $C P$-violation as well as $C P$ conservation hypothesis at $1 \sigma$ C.L. as shown by the green
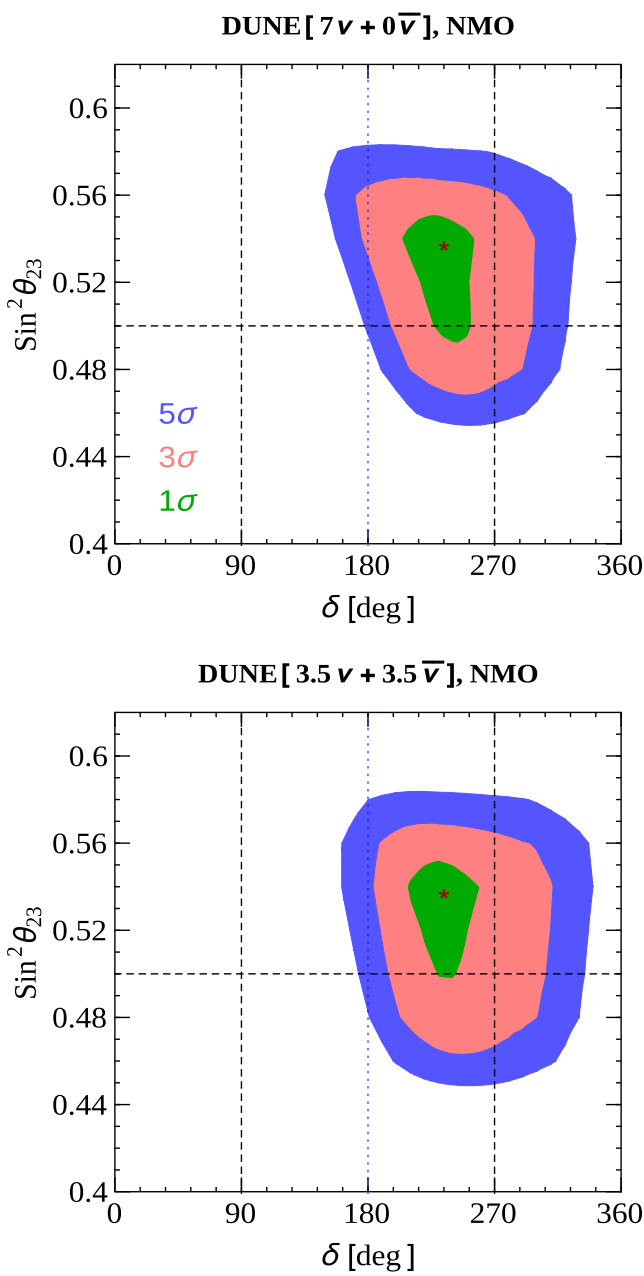

contour for NMO. On the other hand, it cannot exclude either of these hypotheses at $3 \sigma$ C.L. as can be seen from the pink contour which intersects with $\delta=180^{\circ}$ vertical blue-dotted line and $\delta=270^{\circ}$ vertical black-dashed line. Investigating bottom row for normal mass ordering, we notice from first plot that DUNE can exclude maximal $\mathrm{CP}$ violation at $1 \sigma$ C.L. same as only neutrino mode of DUNE. Apart from this it can exclude $C P$-conservation hypothesis at $3 \sigma$ C.L. but not at higher confidence levels.

In the case of IMO, as shown in the right column, we notice that DUNE cannot exclude the phenomenon of maximal $C P$-violation even at $1 \sigma$ C.L. as depicted by the green contour. But, it can exclude $C P$-conservation hypothesis approximately at $5 \sigma$ C.L. as the blue contour marginally touches $\delta=180^{\circ}$. On the other hand, it can reject the $C P$-conservation hypothesis even at $5 \sigma$ C.L. as described by the blue contour of last plot for inverted mass ordering with 3.5 years each of neutrino and antineutrino run of DUNE. Furthermore, normal mass ordering of DUNE $[3.5 \nu+3.5 \bar{\nu}]$ can marginally exclude lower octant (LO)
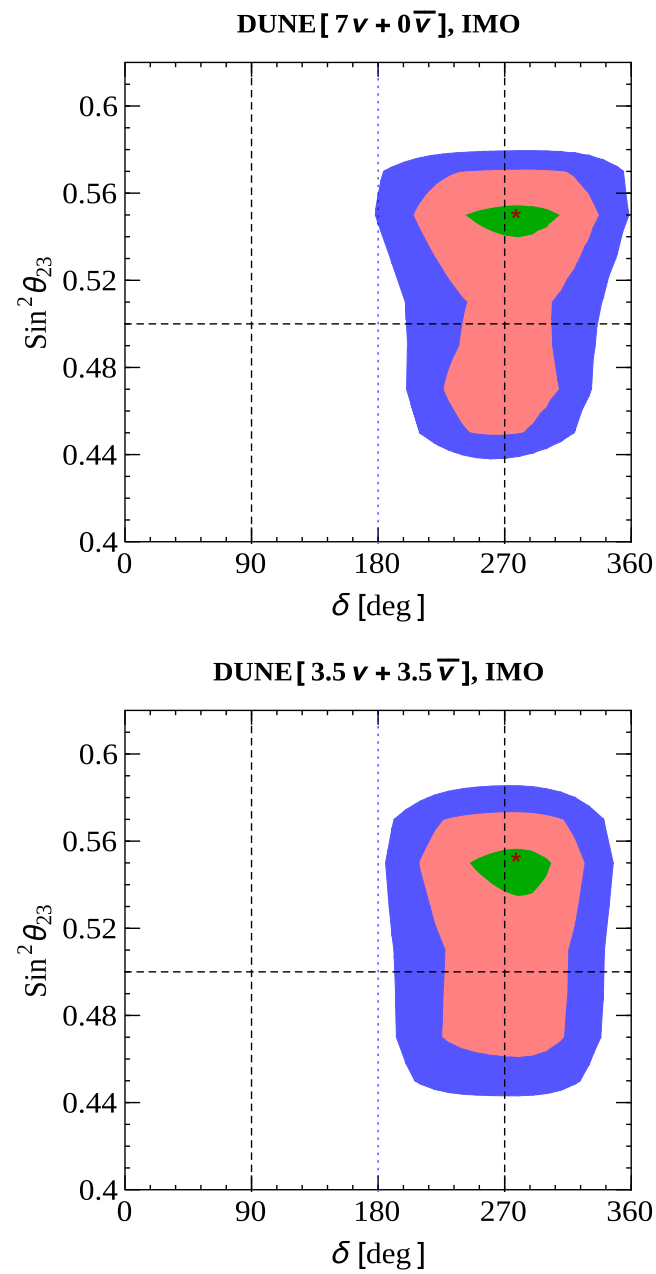

FIG. 2. Allowed parameter space of DUNE in the $\left(\delta-\sin ^{2} \theta_{23}\right)$ plane considering latest NuFit-3.2 data [35]. Remaining details are the same as Fig. 1. 
TABLE III. Modified masses and mixing angles in the BS1 scenario. Here, the second (third) column represents expressions for NMO (INO). Also, we used notation $\varphi_{a c}^{\mu}=\left(\phi_{a}-\phi_{c}+\phi_{\mu}\right), \varphi_{a b}^{\mu}=\left(\phi_{a}+\phi_{b}-\phi_{\mu}\right)$.

\begin{tabular}{lcc}
\hline \hline Parameters $(\mathbf{S 1})$ & $\mathrm{NMO}$ & $\mathrm{IMO}$ \\
\hline$\hat{m}_{1} \simeq$ & 0 & $m_{1}-\epsilon \frac{a}{M_{1}}\left[2 a c_{12}^{2} \cos 2 \phi_{a}\right.$ \\
& $\left.+\sqrt{2} s_{12} c_{12}\left(b \sin \varphi_{a b}^{\mu}-c \sin \varphi_{a c}^{\mu}\right)\right]$ & $m_{2}-\epsilon \frac{a}{M_{1}}\left[2 a s_{12}^{2} \cos 2 \phi_{a}\right.$ \\
$\hat{m}_{2} \simeq$ & $m_{2}-\epsilon \frac{a}{M_{1}}\left[2 a s_{12}^{2} \cos 2 \phi_{a}\right.$ & $\left.+\sqrt{2} s_{12} c_{12}\left(c \sin \varphi_{a c}^{\mu}-b \sin \varphi_{a b}^{\mu}\right)\right]$ \\
& $\left.+\sqrt{2} s_{12} c_{12}\left(c \sin \varphi_{a c}^{\mu}-b \sin \varphi_{a b}^{\mu}\right)\right]$ & 0 \\
$\hat{m}_{3} \simeq$ & $m_{3}-\epsilon \frac{\sqrt{2} a}{M_{1}}\left[b \cos \varphi_{a b}^{\mu}+c \cos \varphi_{a c}^{\mu}\right] \theta_{13}$ & $\theta_{13}+\epsilon \frac{a}{\sqrt{2} m_{2} M_{1}}\left[b \cos \varphi_{a b}^{\mu}+c \cos \varphi_{a c}^{\mu}\right]$ \\
$\hat{\theta}_{13} \simeq$ & $\theta_{13}-\epsilon \frac{a}{\sqrt{2} m_{3} M_{1}}\left[b \cos \varphi_{a b}^{\mu}+c \cos \varphi_{a c}^{\mu}\right]$ & $\theta_{12}-\epsilon \frac{a}{m_{2} M_{1} \xi_{2}}\left[2 a \cos 2 \phi_{a} \sin 2 \theta_{12}\right.$ \\
$\hat{\theta}_{12} \simeq$ & $\theta_{12}-\epsilon \frac{a}{2 m_{3} M_{1} \xi_{1}}\left[2 a \cos 2 \phi_{a} \sin 2 \theta_{12} \cos 2 \theta_{12}\left(c \sin \varphi_{a c}^{\mu}-b \sin \varphi_{a b}^{\mu}\right)\right]$ \\
$\hat{\theta}_{23} \simeq$ & $\left.+\sqrt{2} \cos 2 \theta_{12}\left(c \sin \varphi_{a c}^{\mu}-b \sin \varphi_{a b}^{\mu}\right)\right]$ & $45^{\circ}+\epsilon \frac{a}{\sqrt{2} m_{2} M_{1}}\left[b \cos \varphi_{a b}^{\mu}-c \cos \varphi_{a c}^{\mu}\right] \theta_{13}$ \\
\hline \hline
\end{tabular}

of $\theta_{23}$ (i.e., when $\left.\sin ^{2} \theta_{23} \leq 0.5\right)$ at $1 \sigma$ C.L. as depicted by green contour. Moreover, in the case of IMO, we notice that it can rule out LO of $\theta_{23}$ clearly at $1 \sigma$ C.L. but not at higher confidence levels.

\section{B. Breaking of $\mu-\tau$ reflection symmetry through $M_{D}$}

We discuss here three different scenarios to break $\mu-\tau$ reflection symmetry by introducing explicit breaking parameter in the Dirac neutrino mass matrix, $M_{D}$. Further, for each case we perform precision study to determine $\delta, \sin ^{2} \theta_{23}$ considering DUNE. We study them as follows:

(i) Broken scenario-1 (BS1): After assigning a breaking parameter in the (12) position of $M_{D}$, the new Dirac neutrino mass matrix, $\hat{M}_{D}$ can be written as

$$
\hat{M}_{D}=\left(\begin{array}{cc}
a e^{i \phi_{a}} & a(1+\epsilon) e^{-i \phi_{a}} \\
b e^{i \phi_{b}} & c e^{i \phi_{c}} \\
c e^{-i \phi_{c}} & b e^{-i \phi_{b}}
\end{array}\right)
$$

The above texture of $\hat{M}_{D}$ leads to low-energy neutrino mass matrix $\hat{M}_{\nu}$ of the form,

$$
\begin{aligned}
\hat{M}_{\nu} \simeq & M_{\nu}-\epsilon \frac{a e^{-i \phi_{a}}}{M_{1}}\left(\begin{array}{ccc}
2 a e^{-i \phi_{a}} & c e^{i \phi_{c}} & b e^{-i \phi_{b}} \\
c e^{i \phi_{c}} & 0 & 0 \\
b e^{-i \phi_{b}} & 0 & 0
\end{array}\right) \\
& +\mathcal{O}\left(\epsilon^{2}\right) .
\end{aligned}
$$

Now to find masses and mixing angles in presence of breaking term $\epsilon$, we diagonalize $\hat{M}_{\nu}$ with $\hat{V}$. Note that $\hat{V}$ has similar form as $V$ in the absence of $\epsilon$ as described by Eq. (5). In Table III, we give the expressions of modified masses and mixing angles

\footnotetext{
${ }^{7}$ We vary the breaking term $\epsilon$ in the range, $[-1,1]$ along with other high energy parameters, as mentioned in Eq. (11).
}

for both the mass orderings. Note that for simplicity, we only consider the leading order corrections in terms of $\epsilon, \theta_{13}$ and $\xi_{1}=m_{2} / m_{3}\left(\xi_{2}=\Delta m_{21}^{2} / m_{2}^{2}\right)$ for NMO (IMO).

Afterwards, we proceed to find the set of neutrino oscillation parameters numerically in this scenario. We also emphasize here that the numerical analysis throughout this work are based on exact formula not on any leading order approximations. The numerical best-fit values at $\chi_{\min }^{2}$ for both the mass orderings are tabulated in Table IV. Considering this set of values as the true benchmark point, we present allowed area in the test $\left(\delta-\sin ^{2} \theta_{23}\right)$ plane for DUNE in Fig. $3 .^{8}$

From first plot of Fig. 3, we notice that in the BS1 scenario, DUNE can exclude the theory of maximal CPV at $3 \sigma$ C.L. (see pink contour) for NMO even only with neutrino run. On the other hand, at $5 \sigma$ C.L., this case is unable to exclude both the concerned hypotheses in neutrino mode. With the combined equal neutrino and antineutrino mode analysis of DUNE, we observe that it can exclude the possibility of maximal CPV hypothesis at $3 \sigma$ C.L., whereas $C P$-precision becomes poorer at $5 \sigma$ C.L. as shown in the first plot of the second row. We also notice from both the plots of first column that as the best-fit value of $\delta$ is marginally away from $C P$ conserving value (i.e., $\delta=360^{\circ}$ ), this scenario cannot exclude $C P$-conservation hypothesis even at $1 \sigma$ C.L. In the case of IMO, considering the bestfit values as given by third column of Table IV as the benchmark point, we notice that DUNE can exclude the phenomenon of $C P$-conservation at $3 \sigma$ C.L. but not at $5 \sigma$ C.L. which is depicted in first plot of

\footnotetext{
${ }^{8}$ Note that one can also perform various correlations study considering neutrino oscillation parameters in different broken scenarios. Recently, authors in Ref. [29] have performed different correlations study.
} 
TABLE IV. Set of neutrino oscillation parameters corresponding to $\chi_{\min }^{2}=0.71(=4.5)$ for NMO (IMO) in the BS1 scenario.

\begin{tabular}{lcc}
\hline \hline Parameters & NMO $\left(\chi_{\min }^{2}=0.71\right)$ & IMO $\left(\chi_{\min }^{2}=4.5\right)$ \\
\hline$\Delta \mathrm{m}_{21}^{2}\left[10^{-5} \mathrm{eV}^{2}\right]$ & 7.34 & 7.56 \\
$\left|\Delta \mathrm{m}_{31}^{2}\right|\left[10^{-3} \mathrm{eV}^{2}\right]$ & 2.49 & 2.47 \\
$\sin ^{2} \theta_{12}$ & 0.312 & 0.310 \\
$\sin ^{2} \theta_{23}$ & 0.514 & 0.499 \\
$\sin ^{2} \theta_{13}$ & 0.02235 & 0.02228 \\
$\delta[\mathrm{deg}]$ & 350 & 65 \\
\hline \hline
\end{tabular}

second column by the pink contour. From second plot of right column, we observe that DUNE can reject $C P$-conservation hypothesis even at $5 \sigma$ C.L. Further, both the cases of IMO cannot reject the value corresponding to maximal $\mathrm{CPV}$ even at $1 \sigma$ C.L. We also notice that precision of $\delta$ improves significantly when one chooses IMO over NMO and it gets even better with the combined mode of DUNE run as shown in the last plot. Finally, here we point out that DUNE can exclude $\delta$ in the range,
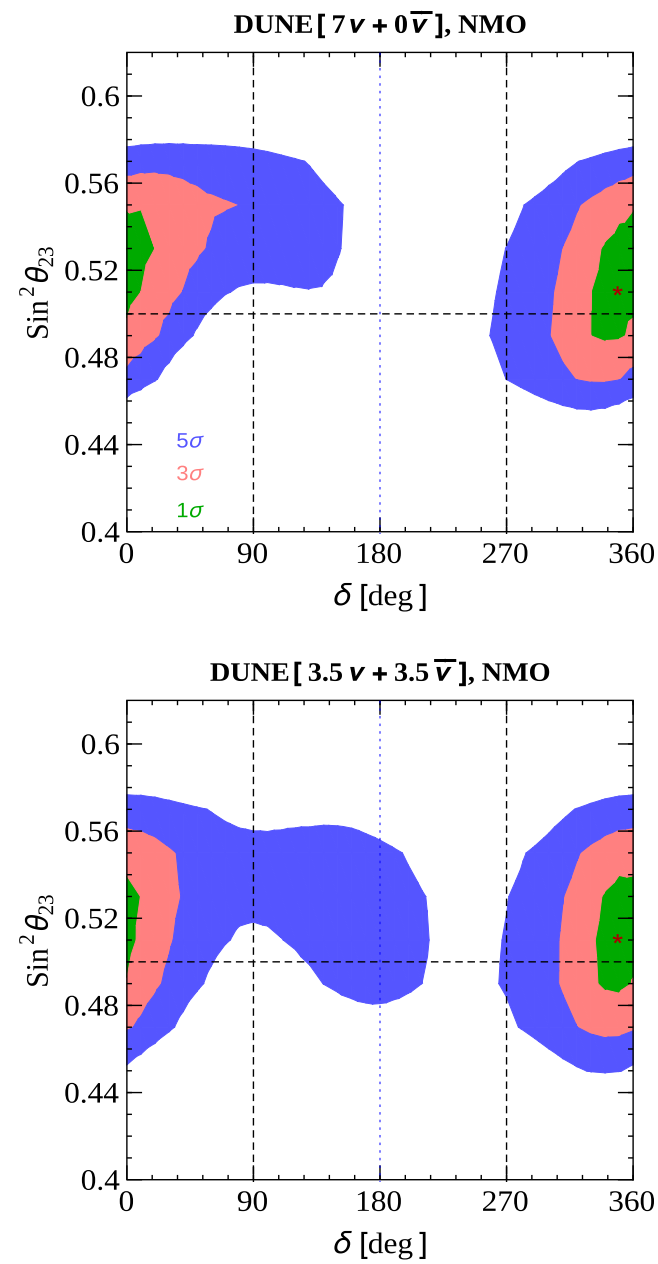

$\delta \in\left[180^{\circ}, 360^{\circ}\right]$ at $3 \sigma$ C.L. for IMO (see first plot of right column), whereas the same conclusion remains permissible even at $5 \sigma$ C.L. with combined $(\nu+\bar{\nu})$ analysis of DUNE (see second plot of right column).

(ii) Broken scenario-2 (BS2): In this scenario, we introduce the breaking term $\epsilon$ in the (22) position of $M_{D}$ and this modifies $M_{D}$ (which we renamed as $\hat{M}_{D}$ ) as

$$
\hat{M}_{D}=\left(\begin{array}{cc}
a e^{i \phi_{a}} & a e^{-i \phi_{a}} \\
b e^{i \phi_{b}} & c(1+\epsilon) e^{i \phi_{c}} \\
c e^{-i \phi_{c}} & b e^{-i \phi_{b}}
\end{array}\right) .
$$

Using the form of $\hat{M}_{D}$ as given by Eq. (15), we find modified $\hat{M}_{\nu}$ as,

$$
\begin{aligned}
\hat{M}_{\nu} \simeq & M_{\nu}-\epsilon \frac{c e^{i \phi_{c}}}{M_{1}}\left(\begin{array}{ccc}
0 & a e^{-i \phi_{a}} & 0 \\
a e^{-i \phi_{a}} & 2 c e^{i \phi_{c}} & b e^{-i \phi_{b}} \\
0 & b e^{-i \phi_{b}} & 0
\end{array}\right) \\
& +\mathcal{O}\left(\epsilon^{2}\right) .
\end{aligned}
$$
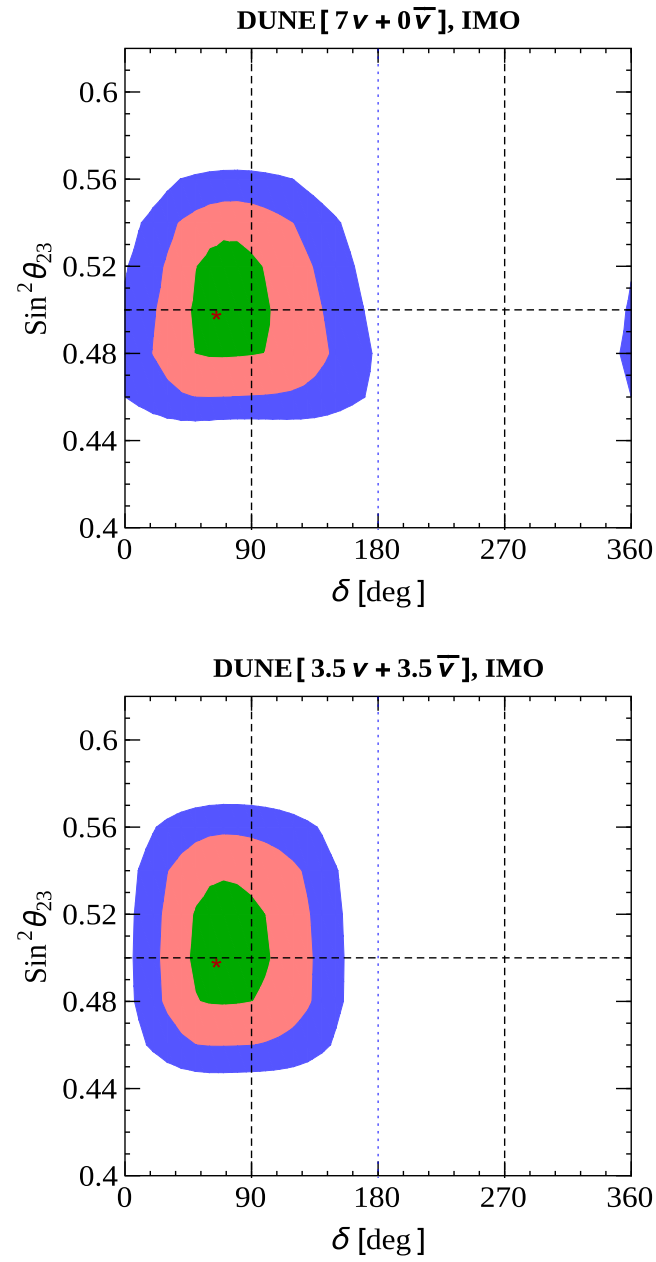

FIG. 3. Allowed parameter space of DUNE in the $\left(\delta-\sin ^{2} \theta_{23}\right)$ plane in the BS1 scenario. Here green, pink, and blue colors represent $1 \sigma, 3 \sigma$, and $5 \sigma$ allowed contours and 'red-*' signifies true value of $\left(\delta, \sin ^{2} \theta_{23}\right)$. 
TABLE V. Modified masses and mixing angles in the BS2 scenario. Notation adopted here are same as Eq. (7) and Table III.

\begin{tabular}{lcc}
\hline \hline Parameters $(\mathbf{S 2})$ & $\mathrm{NMO}$ & $\mathrm{IMO}$ \\
\hline$\hat{m}_{1} \simeq$ & 0 & $m_{1}+\epsilon \frac{c}{M_{1}}\left[\sqrt{2} a s_{12}^{2} \sin \varphi_{a c}^{\mu}\right.$ \\
& & $\left.+b s_{12} c_{12} \sin \varphi_{a c}^{\mu}+s_{12}^{2} \cos 2\left(\phi_{c}-\phi_{\mu}\right)\right]$ \\
$\hat{m}_{2} \simeq$ & $m_{2}+\epsilon \frac{c}{M_{1}}\left[-\sqrt{2} a s_{12} c_{12} \sin \varphi_{a c}^{\mu}\right.$ \\
& $m_{2}+\epsilon \frac{c}{M_{1}}\left[-\sqrt{2} a s_{12} c_{12} \sin \varphi_{a c}^{\mu}\right.$ & $\left.+c_{12}^{2}\left(c \cos 2\left(\phi_{c}-\phi_{\mu}\right)-b \cos \varphi_{b c}\right)\right]$ \\
$\hat{m}_{3} \simeq$ & $\left.+c_{12}^{2}\left(c \cos 2\left(\phi_{c}-\phi_{\mu}\right)-b \cos \varphi_{b c}\right)\right]$ & 0 \\
$\hat{\theta}_{13} \simeq$ & $m_{3}-\epsilon \frac{c}{M_{1}}\left[b \cos \varphi_{b c}+c \cos 2\left(\phi_{c}-\phi_{\mu}\right)\right]$ & $\theta_{13}+\epsilon \frac{a c}{\sqrt{2} m_{2} M_{1}} \cos \varphi_{a c}^{\mu}$ \\
$\hat{\theta}_{12} \simeq$ & $\theta_{13}-\epsilon \frac{a c}{\sqrt{2} m_{3} M_{1}} \cos \varphi_{a c}^{\mu}$ & $\theta_{12}-\epsilon \frac{c}{m_{2} M_{1} \xi_{2}}\left[c\left(\cos 2\left(\phi_{c}-\phi_{\mu}\right)-b \cos \phi_{b c}\right)\right.$ \\
$\hat{\theta}_{23} \simeq$ & $\theta_{12}-\epsilon \frac{c}{2 m_{3} M_{1} \xi_{1}}\left[c\left(\cos 2\left(\phi_{c}-\phi_{\mu}\right)-b \cos \phi_{b c}\right)\right.$ & $\left.\times \sin 2 \theta_{12}+\sqrt{2} a \cos 2 \theta_{12} \sin \varphi_{a c}^{\mu}\right]$ \\
\hline \hline
\end{tabular}

To find modified masses and mixing angles in the given scenario, we follow the similar steps as discussed in subsection III B. In the following Table V, we give their expressions for both the mass orderings. The subleading order term in $\epsilon$ shows the corrections in active neutrino masses and mixing angles for this broken pattern.

After finding analytical expressions, we now evaluate the numerical set of neutrino oscillation parameters in the broken scenario BS2. We calculate the best-fit values corresponding to $\chi_{\min }^{2}$ numerically and present them in Table VI.

Moreover, considering the given set of values as our true benchmark point, we show allowed parameter space of DUNE in the $\left(\delta-\sin ^{2} \theta_{23}\right)$ plane for NMO as well as IMO in Fig. 4. In the case of NMO, as described in left panel, we notice that as the concerned scenario predicts, $\delta=89^{\circ}$ and $\sin ^{2} \theta_{23}=0.49$ neutrino mode of DUNE cannot rule out maximal CPV hypothesis even at $1 \sigma$ C.L. This observation prolongs things further, even with the inclusion of the antineutrino run with the neutrino as depicted by the second plot of the first panel, whereas the neutrino mode of it can exclude the $C P$-conservation hypothesis at $3 \sigma$. In addition, the combined $(\nu+\bar{\nu})$ run can reject the same scenario approximately at $5 \sigma$ C.L. as shown by the blue contour. We also notice by comparing both the plots of the left column that

TABLE VI. Set of neutrino oscillation parameters corresponding to $\chi_{\min }^{2}=1.01(=4.58)$ for NMO (IMO) in the BS2 scenario.

\begin{tabular}{lcc}
\hline \hline Parameters & NMO $\left(\chi_{\min }^{2}=1.01\right)$ & $\mathrm{IMO}\left(\chi_{\min }^{2}=4.58\right)$ \\
\hline$\Delta \mathrm{m}_{21}^{2}\left[10^{-5} \mathrm{eV}^{2}\right]$ & 7.428 & 7.56 \\
$\left|\Delta \mathrm{m}_{31}^{2}\right|\left[10^{-3} \mathrm{eV}^{2}\right]$ & 2.499 & 2.450 \\
$\sin ^{2} \theta_{12}$ & 0.305 & 0.301 \\
$\sin ^{2} \theta_{23}$ & 0.49 & 0.51 \\
$\sin ^{2} \theta_{13}$ & 0.0218 & 0.0229 \\
$\delta[\mathrm{deg}]$ & 89 & 125 \\
\hline \hline
\end{tabular}

DUNE can reject $\delta$ in the range $\delta \in\left[180^{\circ}, 360^{\circ}\right]$ at $3 \sigma$, $5 \sigma$ C.L. considering only neutrino and combined $(\nu+\bar{\nu})$ mode run of DUNE, respectively. From right panel (which is for IMO), we find that both cases can rule out maximal $\mathrm{CPV}$ as well as $C P$-conservation hypothesis only at $1 \sigma$ C.L. Besides this, we notice that only neutrino mode data of DUNE can exclude the $C P$-conservation hypothesis at $3 \sigma$ C.L. but not at $5 \sigma$ C.L., whereas the combined effect of $(\nu+\bar{\nu})$ can reject the same hypothesis at $5 \sigma$ C.L. as shown in the bottom right panel by the blue contour. At the end, we notice from the right panel that $C P$ precision improves significantly with the combined effect of the neutrino and antineutrino run for DUNE, and it can successfully exclude $\delta$ in the range $\delta \in\left[180^{\circ}, 360^{\circ}\right]$ at $5 \sigma$ C.L. In addition, comparing both the columns, we find here that NMO shows better $C P$ precision over IMO.

(iii) Broken scenario-3 (BS3): Here, we assign the breaking parameter in the (32) position of $M_{D}$, and we write the new Dirac neutrino mass matrix, $\hat{M}_{D}$ as

$$
\hat{M}_{D}=\left(\begin{array}{cc}
a e^{i \phi_{a}} & a e^{-i \phi_{a}} \\
b e^{i \phi_{b}} & c e^{i \phi_{c}} \\
c e^{-i \phi_{c}} & b(1+\epsilon) e^{-i \phi_{b}}
\end{array}\right)
$$

$\hat{M}_{D}$ given by Eq. (17), leads us to the following $\hat{M}_{\nu}$ through type-I seesaw formalism,

$$
\begin{aligned}
\hat{M}_{\nu} \simeq M_{\nu}-\epsilon \frac{b e^{-i \phi_{b}}}{M_{1}} & \left(\begin{array}{ccc}
0 & 0 & a e^{-i \phi_{a}} \\
0 & 0 & c e^{i \phi_{c}} \\
b e^{-i \phi_{a}} & c e^{i \phi_{c}} & 2 b e^{-2 i \phi_{b}}
\end{array}\right) \\
& +\mathcal{O}\left(\epsilon^{2}\right) .
\end{aligned}
$$

Now we diagonalize $\hat{M}_{\nu}$ as given by Eq. (18) to find corrections in masses and mixing angles. Here also we perform similar study as discussed in subsection III B. 

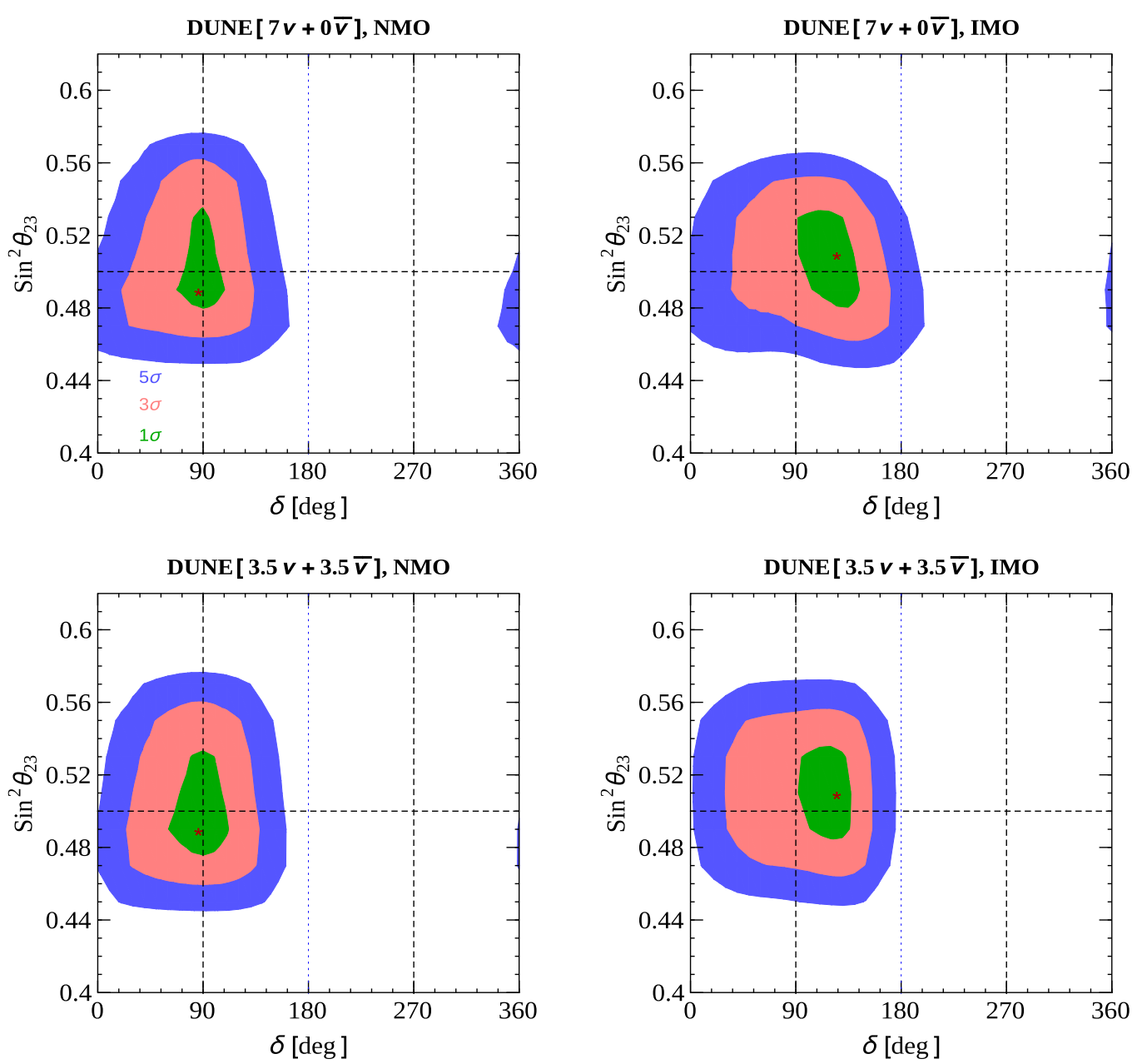

FIG. 4. Allowed parameter space of DUNE in the $\left(\delta-\sin ^{2} \theta_{23}\right)$ plane in the BS2 scenario. Here green, pink, and blue colors represent $1 \sigma, 3 \sigma$ and $5 \sigma$ allowed contours and red-* signifies true value of $\left(\delta, \sin ^{2} \theta_{23}\right)$.

In Table VII, we give analytical expressions for masses and mixing angles considering both the mass orderings where $\mathcal{O}(\epsilon)$ term shows the correction in active neutrino masses and mixing angles for the concerned scenario.
Having discussed analytical results, we proceed to find the set of neutrino oscillation parameters in the broken scenario BS3. We calculate the best-fit values corresponding to $\chi_{\min }^{2}$ numerically and present them in Table VIII. Using this set of true benchmark points,

TABLE VII. Modified masses and mixing angles in the BS3 scenario. Notation adopted here are same as Eq. (7) and Table III.

\begin{tabular}{lcc}
\hline \hline Parameters $(\mathbf{S 3})$ & $\mathrm{NMO}$ & $\mathrm{IMO}$ \\
\hline$\hat{m}_{1} \simeq$ & 0 & $m_{2}+\epsilon \frac{b}{M_{1}}\left[-\sqrt{2} a s_{12} c_{12} \sin \varphi_{a b}^{\mu}\right.$ \\
& & $\left.+c_{12}^{2}\left(b \cos 2\left(\phi_{b}-\phi_{\mu}\right)-c \cos \varphi_{b c}\right)\right]$ \\
$\hat{m}_{2} \simeq$ & $m_{2}+\epsilon \frac{b}{M_{1}}\left[\sqrt{2} a s_{12} c_{12} \sin \varphi_{a b}^{\mu}\right.$ \\
& $m_{2}+\epsilon \frac{b}{M_{1}}\left[\sqrt{2} a s_{12} c_{12} \sin \varphi_{a b}^{\mu}\right.$ & $\left.+c_{12}^{2}\left(b \cos 2\left(\phi_{b}-\phi_{\mu}\right)-c \cos \varphi_{b c}\right)\right]$ \\
$\hat{m}_{3} \simeq$ & $\left.+c_{12}^{2}\left(b \cos 2\left(\phi_{b}-\phi_{\mu}\right)-c \cos \varphi_{b c}\right)\right]$ & 0 \\
$\hat{\theta}_{13} \simeq$ & $m_{3}-\epsilon \frac{b}{M_{1}}\left[b \cos 2\left(\phi_{b}-\phi_{\mu}\right)+c \cos \varphi_{b c}\right]$ & $\theta_{13}+\epsilon \frac{a b}{\sqrt{2} m_{2} M_{1}} \cos \varphi_{a b}^{\mu}$ \\
$\hat{\theta}_{12} \simeq$ & $\theta_{13}-\epsilon \frac{a b}{\sqrt{2} m_{3} M_{1}} \cos \varphi_{a b}^{\mu}$ & $\theta_{12}+\epsilon \frac{b}{m_{2} M_{1} \xi_{2}}\left[\left(c \cos \phi_{b c}-b \cos 2\left(\phi_{b}-\phi_{\mu}\right)\right) \sin 2 \theta_{12}\right.$ \\
& $\theta_{12}+\epsilon \frac{b}{2 m_{3} M_{1} \xi_{1}}\left[\left(c \cos \phi_{b c}-b \cos 2\left(\phi_{b}-\phi_{\mu}\right)\right) \sin 2 \theta_{12}\right.$ & $\left.+\sqrt{2} a \cos 2 \theta_{12} \sin \varphi_{a b}^{\mu}\right]$ \\
$\hat{\theta}_{23} \simeq$ & $\left.+\sqrt{2} a \cos 2 \theta_{12} \sin \varphi_{a b}^{\mu}\right]$ & $45^{\circ}+\epsilon \frac{b^{2}}{m_{2} M_{1}} \cos 2\left(\phi_{b}-\phi_{\mu}\right)$ \\
\hline \hline
\end{tabular}


TABLE VIII. Set of neutrino oscillation parameters corresponding to $\chi_{\min }^{2}=0.62(=5.39)$ for NMO (IMO) in the BS3 scenario.

\begin{tabular}{lcc}
\hline \hline Parameters & NMO $\left(\chi_{\min }^{2}=0.62\right)$ & IMO $\left(\chi_{\min }^{2}=5.39\right)$ \\
\hline$\Delta \mathrm{m}_{21}^{2}\left[10^{-5} \mathrm{eV}^{2}\right]$ & 7.49 & 7.28 \\
$\left|\Delta \mathrm{m}_{31}^{2}\right|\left[10^{-3} \mathrm{eV}^{2}\right]$ & 2.493 & 2.428 \\
$\sin ^{2} \theta_{12}$ & 0.311 & 0.316 \\
$\sin ^{2} \theta_{23}$ & 0.56 & 0.51 \\
$\sin ^{2} \theta_{13}$ & 0.0219 & 0.0229 \\
$\delta[\operatorname{deg}]$ & 252 & 140 \\
\hline \hline
\end{tabular}

we examine allowed parameter space of DUNE in the $\left(\delta-\sin ^{2} \theta_{23}\right)$ plane for both the mass orderings as shown in Fig. 5 (see figure caption for the adopted color convention and other minutes details).

We observe from first plot of left panel that DUNE with only neutrino mode data is not able to exclude the phenomenon of maximal CPV even at $1 \sigma$ C.L. (see green contour for NMO), whereas it can exclude the
$C P$-conservation hypothesis at $3 \sigma$ C.L. (see pink contour for NMO) but not at $5 \sigma$ C.L. as the blue contour intersects with the blue-dotted vertical line. We find that the similar conclusion remains permissible for the combined effect of $(\nu+\bar{\nu})$ run of DUNE as shown in first plot of second row. In the case of IMO with 7-years neutrino run, we find that DUNE can reject both the concerned hypotheses at $1 \sigma$ C.L. At higher confidence levels, however, it fails to rule out any of these hypotheses as depicted in the first plot of the second panel. Investigating the right-hand side plot of second row, we notice that at $1 \sigma$ C.L. it shows similar behaviour as neutrino mode whereas at $3 \sigma$ C.L. it is able to rule out $C P$-conservation hypothesis but not maximal CPV as shown by the pink contour. Finally, we observe a noteworthy outcome in this scenario compared to the former two breaking patterns - this scenario can exclude the lower octant of $\theta_{23}$ at $1 \sigma$ C.L. for NMO even with 7-years of neutrino mode data of DUNE.
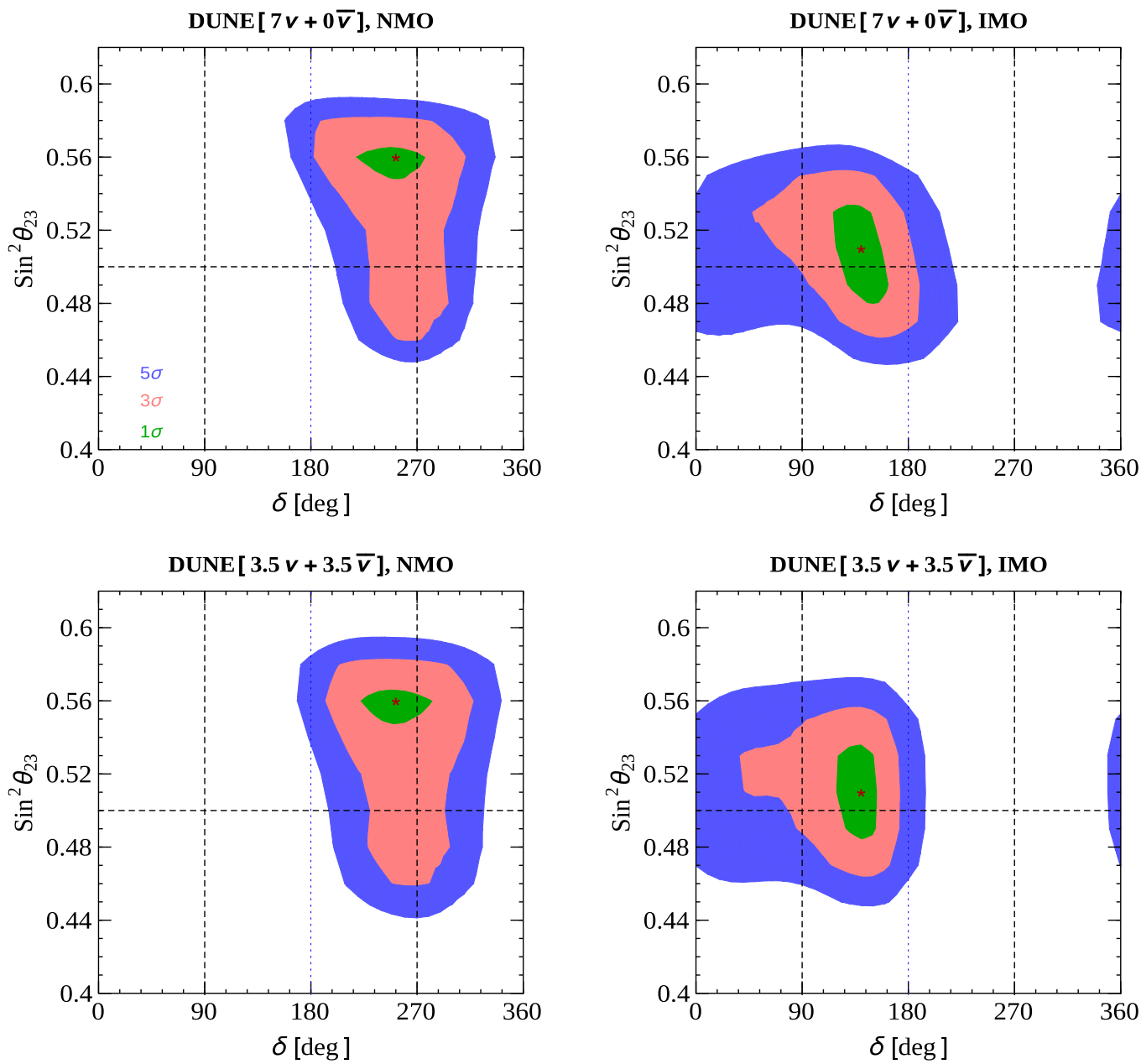

FIG. 5. Allowed parameter space of DUNE in the $\left(\delta-\sin ^{2} \theta_{23}\right)$ plane in the BS3 scenario. Here, green, pink, and blue colors represent $1 \sigma, 3 \sigma$ and $5 \sigma$ allowed contours and red- $*$ signifies true value of $\left(\delta, \sin ^{2} \theta_{23}\right)$. 

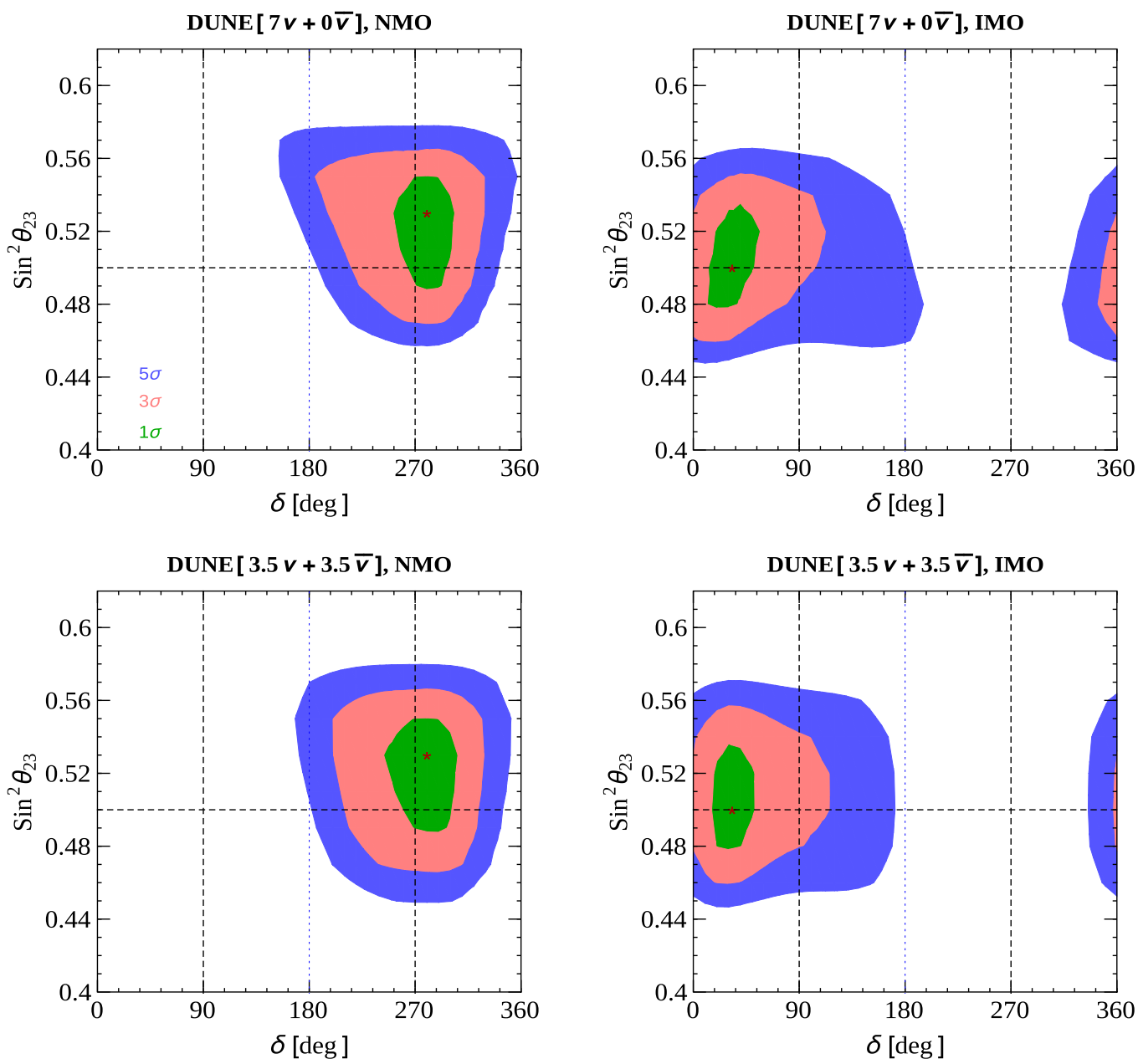

FIG. 6. Allowed parameter space of DUNE in the $\left(\delta-\sin ^{2} \theta_{23}\right)$ plane for the BS4 scenario. Remaining details are same as Fig. 1.

\section{Breaking of $\mu-\tau$ reflection symmetry through $M_{R}$}

We discuss here the breaking of $\mu-\tau$ reflection symmetry by introducing explicit breaking parameter in the Majorana neutrino mass matrix, $M_{R}$. We discuss the scenario as below.

(i) Broken scenario-4 (BS4): After assigning the breaking parameter in the (22) position of $M_{R}$, the modified Majorana neutrino mass matrix, $\hat{M}_{R}$ becomes

$$
\hat{M}_{R}=\left(\begin{array}{cc}
M_{1} & 0 \\
0 & M_{1}(1+\epsilon)
\end{array}\right) .
$$

Note here that, in this scenario, $\hat{M}_{R}$ becomes nondegenerate. After integrating out heavy right-handed neutrino fields, the low-energy neutrino mass matrix in the type-I seesaw formalism can be written as

$$
\begin{aligned}
\hat{M}_{\nu} \simeq & M_{\nu} \\
& -\frac{\epsilon}{M_{1}}\left(\begin{array}{ccc}
a^{2} e^{-2 i \phi_{a}} & a c e^{-i\left(\phi_{a}-\phi_{c}\right)} & a b e^{-i\left(\phi_{a}+\phi_{b}\right)} \\
- & b^{2} e^{-2 i \phi_{b}} & b c e^{-i\left(\phi_{b}-\phi_{c}\right)} \\
- & - & c^{2} e^{-2 i \phi_{c}}
\end{array}\right) \\
& +\mathcal{O}\left(\epsilon^{2}\right) .
\end{aligned}
$$

In this framework, we notice from Eq. (20) that as all the entries of $\mathcal{O}(\epsilon)$ term are nonzero. Therefore, it is highly nontrivial to perform analytical study and to find expressions for modified neutrino masses and mixing angles. Thus, we proceed to employ only numerical study unlike previous subsections, where both analytical as well as numerical study was performed. The set of neutrino oscillation parameters at $\chi_{\min }^{2}$ for possible mass ordering is tabulated in Table IX. We notice from the table that best-fit values corresponding to $\chi_{\text {min }}^{2}$ deviates from maximal $\left(\delta, \theta_{23}\right)$ for $\mathrm{NMO}$ whereas for IMO the given mass textures still favor maximal $\theta_{23}$ but not maximal $\delta$. 
TABLE IX. Set of neutrino oscillation parameters corresponding to $\chi_{\min }^{2}=0.53(=3.91)$ for NMO (IMO) in the BS4 scenario.

\begin{tabular}{lcc}
\hline \hline Parameters & NMO $\left(\chi_{\min }^{2}=0.53\right)$ & IMO $\left(\chi_{\min }^{2}=3.91\right)$ \\
\hline$\Delta \mathrm{m}_{21}^{2}\left[10^{-5} \mathrm{eV}^{2}\right]$ & 7.31 & 7.38 \\
$\left|\Delta \mathrm{m}_{31}^{2}\right|\left[10^{-3} \mathrm{eV}^{2}\right]$ & 2.497 & 2.456 \\
$\sin ^{2} \theta_{12}$ & 0.302 & 0.303 \\
$\sin ^{2} \theta_{23}$ & 0.53 & 0.50 \\
$\sin ^{2} \theta_{13}$ & 0.02179 & 0.02228 \\
$\delta[\mathrm{deg}]$ & 280 & 33 \\
\hline \hline
\end{tabular}

After finding the set of best-fit values at $\chi_{\min }^{2}$, we proceed to analyze its impact on DUNE. Performing similar kinds of analysis as illustrated in the former broken scenarios, we also show here the allowed parameter space of DUNE considering two poorly determined parameters, viz, $\delta$ and $\sin ^{2} \theta_{23}$. We show our results in Fig. 6 considering the test $\left(\delta-\sin ^{2} \theta_{23}\right)$ plane. Now from both the plots of first column, we notice that as the given mass textures have chosen the value of Dirac $C P$-phase, $\delta$ slightly away from its maximal value at $\chi_{\min }^{2}$, DUNE fails to rule out the phenomenon of maximal CPVeven at $1 \sigma$ C.L. In fact, it can rule out $C P$-conservation hypothesis at $3 \sigma$ C.L. even with only neutrino run as shown in first plot of top row by pink contour. We see similar conclusion from the second plot of first column. We also notice here that DUNE with 3.5 years of each neutrino and antineutrino mode data can approximately exclude $\delta$ in the range, $\delta \in\left[0^{\circ}, 180^{\circ}\right]$ at $5 \sigma$ C.L. for the normal mass ordering. In the case of IMO, as depicted in the right column, we find that DUNE can exclude both the concerned phenomena, viz., maximal CPV and $C P$-conservation at $1 \sigma$ C.L. but not at higher confidence levels. Also, none of the cases are able to rule out lower octant of $\sin ^{2} \theta_{23}$ even at $1 \sigma$ C.L. In addition, we find here that NMO shows better $C P$ precision over IMO.

We add a remark here that as the Majorana neutrino mass matrix is always symmetric, addition of nonzero off-diagonal entry still respect $\mu-\tau$ flavor symmetry and predicts maximal $\delta, \sin ^{2} \theta_{23}$. Hence, here we do not include this as an additional scenario.

We now summarize our results in Table $\mathrm{X}$ for the different scenarios which are depicted in Figs. 1-6. We show the possibility of ruling out maximal $C P$-violation (mCPV) or $C P$-conservation (CPC) hypothesis by check marks $(\checkmark)$ considering DUNE. Whereas if DUNE fails to rule out a concerned hypothesis, we mark this with a cross $(\times)$. Note that the parenthesis in bracket shows our results for CPC hypothesis (see table caption for details).

Finally, we calculate the precisions of the two poorly measured parameter $\delta$ and $\sin ^{2} \theta_{23}$. The precision $(P)$ can be defined as

$$
\begin{aligned}
P(\delta) & =\frac{\delta_{\max }-\delta_{\min }}{360^{\circ}} \times 100 \%, \\
P\left(\sin ^{2} \theta_{23}\right) & =\frac{\left(\sin ^{2} \theta_{23}\right)_{\max }-\left(\sin ^{2} \theta_{23}\right)_{\min }}{\left(\sin ^{2} \theta_{23}\right)_{\max }+\left(\sin ^{2} \theta_{23}\right)_{\min }} \times 100 \% .
\end{aligned}
$$

Here, $\max (\min )$ refers to the maximum (minimum) value of the concerned parameter in a given contour. Also, we

TABLE X. The possibility of ruling out maximal $C P$-violation (mCPV) or $C P$-conservation (CPC) hypothesis for both the mass orderings at different C.L. in the case of DUNE. We denote the concerned hypothesis (i.e., mCPV/CPC) by the $\checkmark(\times)$ mark when DUNE is able (unable) to rule out the given scenario. Also, the parentheses in the bracket show our result for the CPC hypothesis. Note that here

\begin{tabular}{|c|c|c|c|c|c|c|}
\hline \multirow[b]{3}{*}{ Scenarios NMO } & \multicolumn{6}{|c|}{ mCPV (CPC) } \\
\hline & \multicolumn{2}{|c|}{$1 \sigma$} & \multicolumn{2}{|c|}{$3 \sigma$} & \multicolumn{2}{|c|}{$5 \sigma$} \\
\hline & $(7 \nu+0 \bar{\nu})$ & $(3.5 \nu+3.5 \bar{\nu})$ & $(7 \nu+0 \bar{\nu})$ & $(3.5 \nu+3.5 \bar{\nu})$ & $(7 \nu+0 \bar{\nu})$ & $(3.5 \nu+3.5 \bar{\nu})$ \\
\hline$\mu-\tau$ & $\times(\mathcal{})$ & $\times(\checkmark)$ & $\times(\mathcal{})$ & $\times(\checkmark)$ & $x(x)$ & $x(x)$ \\
\hline GF & $\checkmark(\checkmark)$ & $\checkmark(\checkmark)$ & $x(x)$ & $\times(\mathcal{})$ & $x(x)$ & $x(x)$ \\
\hline BS1 & $\sqrt{ }(\times)$ & $\sqrt{ }(\times)$ & $\mathcal{V}(\mathrm{x})$ & $\mathcal{V}(\times)$ & $x(x)$ & $x(x)$ \\
\hline BS2 & $\times(\boldsymbol{\downarrow})$ & $\times(\boldsymbol{\downarrow})$ & $\times(\checkmark)$ & $\times(\sqrt{ })$ & $x(x)$ & $x(x)$ \\
\hline BS3 & $\times(\sqrt{ })$ & $\times(\boldsymbol{})$ & $\times(\mathcal{})$ & $\times(\boldsymbol{})$ & $x(x)$ & $x(x)$ \\
\hline BS4 & $\begin{array}{c}\times(\mathcal{J}) \\
1 \sigma\end{array}$ & $\times(\sqrt{ })$ & $\begin{array}{c}\times(\boldsymbol{}) \\
3 \sigma\end{array}$ & $\times(\boldsymbol{J})$ & $\begin{array}{c}\times(\times) \\
5 \sigma\end{array}$ & $x(x)$ \\
\hline Scenarios IMO & $(7 \nu+0 \bar{\nu})$ & $(3.5 \nu+3.5 \bar{\nu})$ & $(7 \nu+0 \bar{\nu})$ & $(3.5 \nu+3.5 \bar{\nu})$ & $(7 \nu+0 \bar{\nu})$ & $(3.5 \nu+3.5 \bar{\nu})$ \\
\hline$\mu-\tau$ & $\times(\checkmark)$ & $\times(\sqrt{ })$ & $\times(\mathcal{})$ & $\times(\checkmark)$ & $\times(\times)$ & $\times(\boldsymbol{})$ \\
\hline GF & $\times(\sqrt{ })$ & $\times(\sqrt{ })$ & $\times(\boldsymbol{})$ & $\times(\boldsymbol{})$ & $x(x)$ & $\times(\boldsymbol{})$ \\
\hline BS1 & $\times(\mathcal{V})$ & $\times(\boldsymbol{})$ & $\times(\mathcal{})$ & $\times(\mathcal{V})$ & $x(x)$ & $\times(\boldsymbol{})$ \\
\hline BS2 & $\checkmark(\checkmark)$ & $\checkmark(\checkmark)$ & $\times(\boldsymbol{})$ & $\times(\boldsymbol{J})$ & $x(\times)$ & $\times(\boldsymbol{})$ \\
\hline BS3 & $\checkmark(\checkmark)$ & $\checkmark(\checkmark)$ & $x(x)$ & $\times(\mathcal{})$ & $x(x)$ & $\times(\times)$ \\
\hline BS4 & $\checkmark(\checkmark)$ & $\checkmark(\checkmark)$ & $x(x)$ & $\mathcal{V}(\times)$ & $x(\times)$ & $\times(\times)$ \\
\hline
\end{tabular}
" $\mu-\tau$ " refers to the symmetry scenario and the abbreviation "GF" stands for the scenario corresponding to global-fit data. 
present the precision table considering $3 \sigma$ confidence level for all the cases that we have considered here around their true values.

\section{CONCLUSION}

In this paper we present an elaborate discussion on the capability of DUNE experiment to test the consequences of $\mu-\tau$ reflection symmetry considering two different modes namely, (i) 7-years of neutrino run and (ii) 3.5-years each of neutrino and antineutrino run. In addition, to realize $\mu-\tau$ reflection symmetry in the low-energy neutrino mass matrix under minimal type-I seesaw formalism, we add two heavy right-handed neutrino fields in the SM. This symmetry predicts maximal atmospheric mixing angle (i.e., $\theta_{23}=45^{\circ}$ ) and Dirac $C P$ phase (i.e., $\delta= \pm 90^{\circ}$ ) along with trivial Majorana phases in the leptonic sector. In this framework, we also find remaining oscillation parameters both analytically as well as numerically. Later, considering numerical best-fit values of neutrino oscillation parameters as our true benchmark point, we find the allowed area in the $\left(\delta-\sin ^{2} \theta_{23}\right)$ plane for DUNE. Further, as the latest global best-fit data prefer nonmaximal $\delta$ as well as $\theta_{23}$, we perform our study considering global best-fit values as one of our true benchmark point in the context of DUNE. Subsequently, we extend our study to break $\mu-\tau$ reflection symmetry by introducing explicit breaking term in the high energy Dirac and Majorana neutrino mass matrices, respectively. Given the breaking scenario, we calculate the set of neutrino oscillation parameters and considering this set as the true benchmark point, we find the allowed area in the test $\left(\delta-\sin ^{2} \theta_{23}\right)$ plane for DUNE. It is noteworthy to make a note here that allowed parameter space in the test $\left(\delta-\sin ^{2} \theta_{23}\right)$ plane also gives an idea about the precision of these two poorly determined parameters for DUNE. Later, we examine the potential of DUNE to rule out maximal $C P$-violation (CPV) or $C P$-conservation hypothesis in each broken scenario.

We summarize DUNE's capability to test interesting hypotheses for all considered cases in Table X. Given the framework of $\mu-\tau$ reflection symmetry, we notice that
DUNE can rule out $C P$-conservation hypothesis at $3 \sigma$ confidence level even with only the neutrino mode run for both the mass orderings, respectively, whereas the DUNE $[3.5 \nu+3.5 \bar{\nu}]$ mode can reject the same at $5 \sigma$ only in the case of IMO. Further, considering global best-fit values as one of our cases, we find that both the considered modes of DUNE can exclude both hypotheses at $1 \sigma$ C.L. only for $\mathrm{NMO}$, whereas it can exclude the $C P$-conservation hypothesis at $5 \sigma$ C.L. for IMO with $(3.5 \nu+3.5 \bar{\nu})$ mode of DUNE but not in the case of NMO. Later, by inspecting broken scenario BS1, we notice that DUNE can exclude the phenomenon of maximal CPV at $3 \sigma$ C.L but not the phenomenon of $C P$-conservation even at $1 \sigma$ C.L. for NMO. Subsequently for IMO, we find that it can rule out $C P$-conservation hypothesis even at $5 \sigma$ C.L. with DUNE $[3.5 \nu+3.5 \bar{\nu}]$ but not maximal CPV hypothesis. Moving to the BS2 scenario, we observe that both the specifications of DUNE can exclude $C P$-conservation hypothesis at $3 \sigma$ C.L. for NMO as well as IMO. Besides this, it can rule out theory of $C P$-conservation even at $5 \sigma$ C.L. only for inverted mass ordering. Examining both the BS3 and BS4 scenarios, we come to the conclusion that DUNE can exclude either the maximal $C P$-violation or $C P$-conservation hypothesis at $1 \sigma$ C.L. for IMO, whereas both of the scenarios can rule out the $C P$-conservation hypothesis at $3 \sigma$ C.L. only for NMO. In the case of IMO, $\mathrm{BS} 3$ can rule out $C P$-conservation hypothesis at $3 \sigma$ C.L., whereas BS4 can exclude the maximal CPV hypothesis at $3 \sigma$ C.L. considering DUNE $[3.5 \nu+3.5 \bar{\nu}]$. In addition, by inspecting all the scenarios for both the mass orderings, we notice that none of the scenarios of NMO can exclude any of the concerned hypotheses at $5 \sigma$ C.L. However, except for the BS3 and BS4, the remaining scenarios of IMO can exclude the $C P$-conservation hypothesis with DUNE $[3.5 \nu+3.5 \bar{\nu}]$ at the same confidence level.

Afterwards, we also examine the precision of both the less-known parameters, $\delta, \theta_{23}$, and as a case study, we present our results at $3 \sigma$ confidence level in Table XI. By scrutinizing all the possibilities, we notice that the BS4 case gives the worst precision on the Dirac $C P$-phase, $\delta$ of $41.1 \%$ in the case of DUNE[7 $7 \nu+0 \bar{\nu}]$ for NMO, whereas

TABLE XI. Precision table of $\delta, \sin ^{2} \theta_{23}$ for all the considered scenarios of $\left(\delta, \sin ^{2} \theta_{23}\right)$ in the case of DUNE[7 $\left.\nu+0 \bar{\nu}\right]$ and DUNE $[3.5 \nu+3.5 \bar{\nu}]$ at $3 \sigma$ C.L.

\begin{tabular}{|c|c|c|c|c|c|c|c|c|}
\hline \multirow[b]{3}{*}{ Scenarios } & \multicolumn{4}{|c|}{ NMO (in \%) } & \multicolumn{4}{|c|}{ IMO (in \%) } \\
\hline & \multicolumn{2}{|c|}{$P(\delta)$} & \multicolumn{2}{|c|}{$P\left(\sin ^{2} \theta_{23}\right)$} & \multicolumn{2}{|c|}{$P(\delta)$} & \multicolumn{2}{|c|}{$P\left(\sin ^{2} \theta_{23}\right)$} \\
\hline & $(7 \nu+0 \bar{\nu})$ & $(3.5 \nu+3.5 \bar{\nu})$ & $(7 \nu+0 \bar{\nu})$ & $(3.5 \nu+3.5 \bar{\nu})$ & $(7 \nu+0 \bar{\nu})$ & $(3.5 \nu+3.5 \bar{\nu})$ & $(7 \nu+0 \bar{\nu})$ & $(3.5 \nu+3.5 \bar{\nu})$ \\
\hline$\mu-\tau$ & 32.5 & 31.9 & 8.7 & 9.3 & 37.5 & 32.2 & 8.7 & 9.1 \\
\hline GF & 36.9 & 34.7 & 9.2 & 9.9 & 35.0 & 31.6 & 11.6 & 10.5 \\
\hline BS1 & 36.1 & 25.0 & 9.2 & 8.9 & 38.8 & 30.0 & 8.9 & 9.4 \\
\hline BS2 & 31.4 & 31.9 & 9.3 & 9.8 & 37.5 & 35.0 & 8.9 & 8.5 \\
\hline BS3 & 34.2 & 32.7 & 11.5 & 11.3 & 40.8 & 36.9 & 8.9 & 8.8 \\
\hline BS4 & 41.1 & 38.6 & 9.0 & 9.3 & 37.5 & 33.8 & 8.9 & 9.4 \\
\hline
\end{tabular}


BS1 comes with the best precision of $25.0 \%$ among all

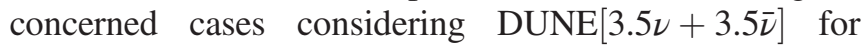
NMO. Similarly, for the 2-3 mixing angle, $\theta_{23}$, we find that global best-fit value with DUNE[ $7 \nu+0 \bar{\nu}]$ mode gives a worst precision of $11.6 \%$ for IMO, whereas BS2 for IMO

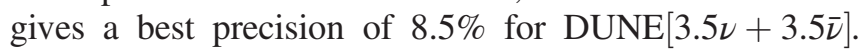
Also, by investigating all scenarios, we notice that the scenario BS3 is able to exclude the lower octant of $\theta_{23}$ at $1 \sigma$ C.L. for NMO and analysis of global best-fit value shows similar conclusion in context of IMO. Note that results discussed here can be used to test DUNE's potential for the discrimination of different scenarios.

Finally, we conclude this work with a remark that with the available data in the neutrino oscillation sector, the $\mu-\tau$ reflection symmetry stands out as one of the finest theoretically favored approaches to study some intriguing aspects of neutrinos. On the other hand, forthcoming facilities, like DUNE with its high statistics and ability to measure $\left(\delta, \theta_{23}\right)$ with high precision, serves as an impeccable experiment to test numerous predictions of different models.

\section{ACKNOWLEDGMENTS}

N. N. is grateful to Professor Zhi-zhong Xing for his insightful comments and careful reading of the manuscript. He also would like to thank Professor Srubabati Goswami, Professor Shun Zhou, Dr. Jue Zhang, Dr. Rahul Srivastava, and Mr. Guo-yuan Huang for useful discussions. The research work of N.N. was supported in part by the National Natural Science Foundation of China under Grant No. 11775231.
[1] C. Patrignani et al. (Particle Data Group), Chin. Phys. C 40, 100001 (2016).

[2] P. Minkowski, Phys. Lett. 67B, 421 (1977).

[3] T. Yanagida, Conf. Proc. C 7902131, 95 (1979).

[4] M. Gell-Mann, P. Ramond, and R. Slansky, Conf. Proc. C 790927, 315 (1979).

[5] R. N. Mohapatra and G. Senjanovic, Phys. Rev. Lett. 44, 912 (1980).

[6] J. Schechter and J. W. F. Valle, Phys. Rev. D 22, 2227 (1980).

[7] G. Altarelli and F. Feruglio, Rev. Mod. Phys. 82, 2701 (2010).

[8] G. Altarelli, F. Feruglio, and L. Merlo, Fortschr. Phys. 61, 507 (2013).

[9] A. Yu. Smirnov, J. Phys. Conf. Ser. 335, 012006 (2011).

[10] H. Ishimori, T. Kobayashi, H. Ohki, Y. Shimizu, H. Okada, and M. Tanimoto, Prog. Theor. Phys. Suppl. 183, 1 (2010).

[11] S. F. King and C. Luhn, Rep. Prog. Phys. 76, 056201 (2013).

[12] P. F. Harrison and W. G. Scott, Phys. Lett. B 547, 219 (2002).

[13] Z.-z. Xing and Z.-h. Zhao, Rep. Prog. Phys. 79, 076201 (2016).

[14] P. M. Ferreira, W. Grimus, L. Lavoura, and P. O. Ludl, J. High Energy Phys. 09 (2012) 128.

[15] R. N. Mohapatra and C. C. Nishi, Phys. Rev. D 86, 073007 (2012).

[16] E. Ma, A. Natale, and O. Popov, Phys. Lett. B 746, 114 (2015).

[17] E. Ma, Phys. Lett. B 752, 198 (2016).

[18] H.-J. He, W. Rodejohann, and X.-J. Xu, Phys. Lett. B 751, 586 (2015).

[19] A. S. Joshipura and K. M. Patel, Phys. Lett. B 749, 159 (2015).

[20] A. S. Joshipura, J. High Energy Phys. 11 (2015) 186.
[21] A. S. Joshipura and N. Nath, Phys. Rev. D 94, 036008 (2016).

[22] C. C. Nishi and B. L. Sánchez-Vega, J. High Energy Phys. 01 (2017) 068.

[23] T. Fukuyama, Prog. Theor. Exp. Phys. 3 033B11 (2017).

[24] Z.-h. Zhao, J. High Energy Phys. 09 (2017) 023.

[25] W. Rodejohann and X.-J. Xu, Phys. Rev. D 96, 055039 (2017).

[26] Z.-C. Liu, C.-X. Yue, and Z.-h. Zhao, J. High Energy Phys. 10 (2017) 102.

[27] Z.-z. Xing, D. Zhang, and J.-y. Zhu, J. High Energy Phys. 11 (2017) 135.

[28] Z.-z. Xing and J.-y. Zhu, Chin. Phys. C 41, 123103 (2017).

[29] N. Nath, Z.-Z. Xing, and J. Zhang, Eur. Phys. J. C 78, 289 (2018).

[30] Z.-h. Zhao, Nucl. Phys. B935, 129 (2018).

[31] K. Chakraborty, K. N. Deepthi, S. Goswami, A. S. Joshipura, and N. Nath, arXiv:1804.02022.

[32] N. Nath, arXiv:1808.05062

[33] W.-1. Guo, Z.-z. Xing, and S. Zhou, Int. J. Mod. Phys. E 16, 1 (2007).

[34] R. Acciarri et al. (DUNE Collaboration), arXiv:1512.06148.

[35] NuFIT 3.2 Collaboration, 2018, http://www.nu-fit.org/.

[36] I. Esteban, M. C. Gonzalez-Garcia, M. Maltoni, I. MartinezSoler, and T. Schwetz, J. High Energy Phys. 01 (2017) 087.

[37] K. Abe et al. (T2K Collaboration), Phys. Rev. Lett. 118, 151801 (2017).

[38] A. Radovic et al. (NOvA Collaboration), http://nova-docdb .fnal.gov/cgi-bin/ShowDocument?docid=25938.

[39] R. de Adelhart Toorop, F. Feruglio, and C. Hagedorn, Phys. Lett. B 703, 447 (2011).

[40] A. D. Hanlon, S.-F. Ge, and W. W. Repko, Phys. Lett. B 729, 185 (2014).

[41] A. D. Hanlon, W. W. Repko, and D. A. Dicus, Adv. High Energy Phys. 2014, 1 (2014). 
[42] K. Bora, D. Borah, and D. Dutta, Phys. Rev. D 96, 075006 (2017).

[43] R. Srivastava, C. A. Ternes, M. Tórtola, and J. W. F. Valle, Phys. Lett. B 778, 459 (2018).

[44] S. K. Agarwalla, S. S. Chatterjee, S. T. Petcov, and A. V. Titov, Eur. Phys. J. C 78, 286 (2018).

[45] S. S. Chatterjee, M. Masud, P. Pasquini, and J. W. F. Valle, Phys. Lett. B 774, 179 (2017).

[46] P. Pasquini, Adv. High Energy Phys. 2018, 1 (2018).

[47] R. Srivastava, C. A. Ternes, M. Tórtola, and J. W. F. Valle, Phys. Rev. D 97, 095025 (2018).

[48] S. T. Petcov and A. V. Titov, Phys. Rev. D 97, 115045 (2018).
[49] F. Feroz and M. P. Hobson, Mon. Not. R. Astron. Soc. 384, 449 (2008).

[50] F. Feroz, M. P. Hobson, and M. Bridges, Mon. Not. R. Astron. Soc. 398, 1601 (2009).

[51] F. Feroz, M. P. Hobson, E. Cameron, and A. N. Pettitt, arXiv:1306.2144.

[52] T. Alion et al. (DUNE Collaboration), arXiv:1606.09550.

[53] P. Huber, M. Lindner, and W. Winter, Comput. Phys. Commun. 167, 195 (2005).

[54] P. Huber, J. Kopp, M. Lindner, M. Rolinec, and W. Winter, Comput. Phys. Commun. 177, 432 (2007).

[55] N. Nath, M. Ghosh, and S. Goswami, Nucl. Phys. B913, 381 (2016). 